\title{
ROLAND BACHER
}

\section{PIERRE DE LA HARPE}

TATIANA NAGNIBEDA

\section{The lattice of integral flows and the lattice of integral cuts on a finite graph}

Bulletin de la S. M. F., tome 125, no 2 (1997), p. 167-198

<http://www.numdam.org/item?id=BSMF_1997_125_2_167_0>

(C) Bulletin de la S. M. F., 1997, tous droits réservés.

L'accès aux archives de la revue «Bulletin de la S. M. F. » (http: //smf.emath.fr/Publications/Bulletin/Presentation.html) implique l'accord avec les conditions générales d'utilisation (http://www.numdam.org/ conditions). Toute utilisation commerciale ou impression systématique est constitutive d'une infraction pénale. Toute copie ou impression de ce fichier doit contenir la présente mention de copyright.

\section{Numdam}




\title{
THE LATTICE OF INTEGRAL FLOWS AND THE LATTICE OF INTEGRAL CUTS ON A FINITE GRAPH
}

\author{
BY \\ Roland BACHER, Pierre DE LA HARPE \\ and Tatiana NAGNIBEDA $(*)$
}

\begin{abstract}
The set of integral flows on a finite graph $\Gamma$ is naturally an integral lattice $\Lambda^{1}(\Gamma)$ in the Euclidean space $\operatorname{Ker}\left(\Delta_{1}\right)$ of harmonic real-valued functions on the edge set of $\Gamma$. Various properties of $\Gamma$ (bipartite character, girth, complexity, separability) are shown to correspond to properties of $\Lambda^{1}(\Gamma)$ (parity, minimal norm, determinant, decomposability). The dual lattice of $\Lambda^{1}(\Gamma)$ is identified to the integral cohomology $H^{1}(\Gamma, \mathbb{Z})$ in $\operatorname{Ker}\left(\Delta_{1}\right)$. Analogous characterizations are shown to hold for the lattice of integral cuts and appropriate properties of the graph (Eulerian character, edge connectivity, complexity, separability).

These lattices have a determinant group which plays for graphs the same role as Jacobians for closed Riemann surfaces. It is then harmonic functions on a graph (with values in an abelian group) which take place of holomorphic mappings.
\end{abstract}

RÉSUMÉ. - Les flots entiers sur un graphe fini $\Gamma$ constituent naturellement un réseau entier $\Lambda^{1}(\Gamma)$ dans l'espace euclidien $\operatorname{Ker}\left(\Delta_{1}\right)$ des fonctions harmoniques à valeurs réelles sur l'ensemble des arêtes de $\Gamma$. On montre l'équivalence de diverses propriétés de $\Gamma$ (caractère biparti, tour de taille, complexité, séparabilité) avec des propriétés convenables de $\Lambda^{1}(\Gamma)$ (parité, norme minimale, déterminant, décomposabilité). Le réseau dual de $\Lambda^{1}(\Gamma)$ est identifié à la cohomologie entière $H^{1}(\Gamma, \mathbb{Z})$, plongée dans $\operatorname{Ker}\left(\Delta_{1}\right)$.

(*) Texte reçu le 5 avril 1996, accepté le 18 janvier 1997.

R. BACHER, Université de Grenoble 1, Institut Fourier, Laboratoire de Mathématiques associé au CNRS, B.P. 74, 38402 Saint-Martin-d'Hères CEDEX (France).

Email: bacher@fourier.grenet.fr

P. De la Harpe, T. Nagnibeda, Section de Mathématiques, Université de Genève, C.P. 240, CH-1211 Genève 24 (Suisse).

Email: Pierre.delaHarpe@math.unige.ch et Tatiana.Nagnibeda@math.unige.ch.

The first author has been partially supported by the Fonds National Suisse de la Recherche Scientifique.

Keywords: Graph, lattice, combinatorial Laplacian, integral flows, cutsets, Jacobian.

AMS classification: $05 \mathrm{C} 38,11 \mathrm{E} 39$.

BULLETIN DE LA SOCIÉTÉ MATHÉMATIQUE DE FRANCE 0037-9484/1997/167/\$5.00

(C) Société mathématique de France 
On montre des traductions analogues pour le réseau des coupures entières et les propriétés convenables du graphe (caractère eulérien, connectivité d'arêtes, complexité, séparabilité).

Ces réseaux ont un groupe déterminant qui joue pour les graphes le même rôle que la jacobienne pour une surface de Riemann close. Ce sont alors les fonctions harmoniques sur un graphe (à valeurs dans un groupe abélien) qui tiennent lieu d'applications holomorphes.

\section{Introduction: The lattices $\Lambda^{\mathbf{1}}(\Gamma)$ and $N^{\mathbf{1}}(\Gamma)$}

Several classical results relate geometrical properties of a finite graph to properties of various matrices or polynomials attached to the graph. For this algebraic graph theory, see among others [Bi1], [BCN], [CDS]. The purpose of this paper is to show that lattices and quadratic forms fit most naturally in this theory. From another point of view, our aim is to show how finite graphs provide numerous examples of positive definite integral lattices.

More precisely, consider a finite graph $\Gamma$ with vertex set $V$ and with edge set $E$ (loops and multiple edges are allowed). Following Serre (see [Ser, $\left.\S 2, \mathrm{n}^{\circ} 1\right]$ ) we introduce the set $\mathbb{E}$ of oriented edges of $\Gamma$ (the cardinality of $\mathbb{E}$ is twice that of $E$ ) and we denote by $\bar{e} \in \mathbb{E}$ the inverse of an oriented edge $e \in \mathbb{E}$. The vertex space $C^{0}(\Gamma, \mathbb{R})$ of all real functions on $V$ and the edge space $C^{1}(\Gamma, \mathbb{R})$ of all functions $g: \mathbb{E} \rightarrow \mathbb{R}$ such that $g(\bar{e})=-g(e)$ for all $e \in \mathbb{E}$ are Euclidean spaces for inner products defined by

$$
\left\langle f_{1}, f_{2}\right\rangle=\sum_{v \in V} f_{1}(v) f_{2}(v), \quad\left\langle g_{1}, g_{2}\right\rangle=\frac{1}{2} \sum_{e \in \mathbb{E}} g_{1}(e) g_{2}(e)
$$

for all $f_{1}, f_{2} \in C^{0}(\Gamma, \mathbb{R})$ and $g_{1}, g_{2} \in C^{1}(\Gamma, \mathbb{R})$. One defines an "exterior differential"

$$
d: C^{0}(\Gamma, \mathbb{R}) \longrightarrow C^{1}(\Gamma, \mathbb{R})
$$

by

$$
(d f)(e)=f\left(e_{+}\right)-f\left(e_{-}\right)
$$

where $e_{+}$and $e_{-}$denote respectively the head and the tail of an oriented edge $e$. The adjoint operator

$$
d^{*}: C^{1}(\Gamma, \mathbb{R}) \longrightarrow C^{0}(\Gamma, \mathbb{R})
$$

is given by

$$
\left(d^{*} g\right)(v)=\sum_{\substack{e \in \mathbb{E} \\ e_{+}=v}} g(e)
$$

and can be viewed as the usual incidence matrix of $\Gamma$.

TOME $125-1997-\mathrm{N}^{\circ} 2$ 
The corresponding Laplacians

$$
\begin{aligned}
& \Delta_{0}=d^{*} d: C^{0}(\Gamma, \mathbb{R}) \longrightarrow C^{0}(\Gamma, \mathbb{R}), \\
& \Delta_{1}=d d^{*}: C^{1}(\Gamma, \mathbb{R}) \longrightarrow C^{1}(\Gamma, \mathbb{R})
\end{aligned}
$$

are given by the familiar formulas

$$
\left(\Delta_{0} f\right)(v)=\operatorname{deg}(v) f(v)-\sum_{\substack{w \in V \\ w \sim v}} f(w)
$$

(where the sum means more precisely $\sum_{e \in \mathbb{E}, e_{+}=v} f\left(e_{-}\right)$) and

$$
\left(\Delta_{1} g\right)(e)=2 g(e)-\sum_{\substack{e^{\prime} \in \mathbb{E} \\ e_{+}^{\prime}=e_{-}}} g\left(e^{\prime}\right)-\sum_{\substack{e^{\prime \prime} \in \mathbb{E} \\ e_{-}^{\prime \prime}=e_{+}}} g\left(e^{\prime \prime}\right) .
$$

One has orthogonal decompositions

$$
\begin{aligned}
& C^{0}(\Gamma, \mathbb{R})=\operatorname{Ker}\left(\Delta_{0}\right) \oplus \operatorname{Im}\left(d^{*}\right), \\
& C^{1}(\Gamma, \mathbb{R})=\operatorname{Ker}\left(\Delta_{1}\right) \oplus \operatorname{Im}(d) .
\end{aligned}
$$

(Short proof for the first equality: if $x$ is an element of $\operatorname{Ker}\left(\Delta_{0}\right)$ then $\left\langle\Delta_{0} x, x\right\rangle=\langle d x, d x\rangle=0$ and $x \in \operatorname{Ker}(d)$; thus $\operatorname{Ker}\left(\Delta_{0}\right)=\operatorname{Ker}(d)$; the equality follows because $\operatorname{Ker}(d)$ is the orthogonal complement of $\operatorname{Im}\left(d^{*}\right)$ in $C^{0}(\Gamma, \mathbb{R})$.)

The kernel $\operatorname{Ker}\left(\Delta_{0}\right)$ is the set of functions $V \rightarrow \mathbb{R}$ which are constant on the connected components of the graph; the space $\operatorname{Ker}\left(\Delta_{1}\right)$ is often called the cycle subspace and $\operatorname{Im}(d)$ the cut subspace of $\Gamma$ (see e.g. [Bi1, Chap. 4]). We rather think of $\operatorname{Ker}\left(\Delta_{0}\right)$ and $\operatorname{Ker}\left(\Delta_{1}\right)$ as spaces of harmonic functions and harmonic one-forms on $\Gamma$ ("forms" in the sense of differential forms on manifolds).

Integral valued functions on $\mathbb{E}$ provide a lattice $C^{1}(\Gamma, \mathbb{Z}) \subset C^{1}(\Gamma, \mathbb{R})$ which is isomorphic to the trivial lattice $\mathbb{Z}^{m} \subset \mathbb{R}^{m}$ (where $m=|E|=\frac{1}{2}|\mathbb{E}|$ ). By intersection with the two factors of the orthogonal decomposition of $C^{1}(\Gamma, \mathbb{R})$, one obtains the lattice of integral flows

$$
\Lambda^{1}(\Gamma)=C^{1}(\Gamma, \mathbb{Z}) \cap \operatorname{Ker}\left(\Delta_{1}\right) \subset \operatorname{Ker}\left(\Delta_{1}\right)
$$

and the lattice of integral cuts

$$
N^{1}(\Gamma)=C^{1}(\Gamma, \mathbb{Z}) \cap \operatorname{Im}(d) \subset \operatorname{Im}(d) .
$$

These are the fundamental objects of our interest in this work. They are clearly integral lattices as $\langle\lambda, \lambda\rangle \in \mathbb{Z}$ for all $\lambda \in \Lambda^{1}(\Gamma)$ (resp. $\lambda \in N^{1}(\Gamma)$ ). 
One may of course define similarly two lattices $\Lambda^{0}(\Gamma) \subset \operatorname{Ker}\left(\Delta_{0}\right)$ and $M^{0}(\Gamma) \subset \operatorname{Im}\left(d^{*}\right)$. But these are of little interest. If $\Gamma$ is connected and has $n$ vertices, it is easy to check that $\Lambda^{0}(\Gamma)$ is the infinite cyclic group generated by the constant function of value 1 , so that $\Lambda^{0}(\Gamma) \subset \operatorname{Ker}\left(\Delta_{0}\right)$ is isomorphic to $\sqrt{n} \mathbb{Z} \subset \mathbb{R}$, and that $M^{0}(\Gamma)$ is the lattice of integral valued functions in the space $\operatorname{Im}\left(d^{*}\right)$ of functions $f: V \rightarrow \mathbb{R}$ such that $\sum_{v \in V} f(x)=0$ (the root lattice of type $A_{n-1}$ ). In general, $\Lambda^{0}(\Gamma)$ and $M^{0}(\Gamma)$ are direct sums of factors corresponding to the connected components of $\Gamma$.

Let us recall some terminology on lattices. Consider a Euclidean space $\mathcal{H}$; the inner product of two vectors $x, y \in \mathcal{H}$ is denoted by $\langle x, y\rangle$. A lattice in $\mathcal{H}$ is a discrete subgroup $\Lambda$ of $\mathcal{H}$ such that the quotient $\mathcal{H} / \Lambda$ is compact. If $\Lambda$ is a lattice in $\mathcal{H}$, its determinant $\operatorname{det}(\Lambda)$ is the square of the volume of a fundamental domain for the action of $\Lambda$ on $\mathcal{H}$ by translations, and its minimal norm is the number

$$
\min (\Lambda)=\min \{\langle\lambda, \lambda\rangle \mid \lambda \in \Lambda, \lambda \neq 0\} .
$$

The dual lattice is

$$
\Lambda^{\sharp}=\{x \in \mathcal{H} \mid\langle x, \lambda\rangle \in \mathbb{Z} \text { for all } \lambda \in \Lambda\}
$$

(it is also called the "polar lattice"). A lattice $\Lambda$ is decomposable if there exists a non trivial orthogonal decomposition $\mathcal{H}=\mathcal{H}_{1} \oplus \mathcal{H}_{2}$ such that $\Lambda=\left(\Lambda \cap \mathcal{H}_{1}\right) \oplus\left(\Lambda \cap \mathcal{H}_{2}\right)$, and indecomposable otherwise.

A lattice $\Lambda$ is integral if $\langle\lambda, \mu\rangle \in \mathbb{Z}$ for all $\lambda, \mu \in \Lambda$, or equivalently if $\Lambda \subset \Lambda^{\sharp}$. An integral lattice $\Lambda$ is even if $\langle\lambda, \lambda\rangle \in 2 \mathbb{Z}$ for all $\lambda \in \Lambda$.

The determinant group of an integral lattice $\Lambda$ is the group $\Lambda^{\sharp} / \Lambda$; it is a finite group of order $\operatorname{det}(\Lambda)$. (Another term for "determinant group" is quotient group.) It comes with a non-degenerate $\mathbb{Q} / \mathbb{Z}$-valued bilinear form

$$
b_{\Lambda}: \Lambda^{\sharp} / \Lambda \times \Lambda^{\sharp} / \Lambda \longrightarrow \mathbb{Q} / \mathbb{Z}
$$

defined by $b_{\Lambda}(x, y)=\langle\lambda, \mu\rangle \bmod \mathbb{Z}$ where $\lambda, \mu \in \Lambda^{\sharp}$ are respectively representants of $x, y \in \Lambda^{\sharp} / \Lambda$. The finite inner product module $\left(\Lambda^{\sharp} / \Lambda, b_{\Lambda}\right)$ is called the discriminant form of the lattice $\Lambda$. In particular one may attach to any lattice $\Lambda$ the Witt class $w(\Lambda)$ of its discriminant form, which is an element of the Witt group $W(\mathbb{Q} / \mathbb{Z})$; for the Witt group $W(\mathbb{Q} / \mathbb{Z})$ see e.g. $\S 5.1$ in [Sch].

There are open problems formulated at the ends of Sections 2, 3 and 9.

It is a pleasure to acknowledge several useful conversations with Marc Burger, Shalom Eliahou, François Jaeger, Michel Kervaire, Edouard Lebeau and Boris Venkov. We are also grateful to Norman Biggs for his interest in our work [Bi3], [Bi4].

TOME $125-1997-\mathrm{N}^{\circ} 2$ 


\section{Some properties of $\Lambda^{\mathbf{1}}(\Gamma)$ and $N^{\mathbf{1}}(\Gamma)$}

Let $\Gamma$ be a graph. If $\Gamma$ is not connected, both $\Lambda^{1}(\Gamma)$ and $N^{1}(\Gamma)$ are orthogonal sums of the contributions of the connected components of $\Gamma$.

We assume from now on that $\Gamma$ is connected.

If $\Gamma$ is a tree, then $\operatorname{Ker}\left(\Delta_{1}\right)=\{0\}$ and the lattice $\Lambda^{1}(\Gamma)$ is trivial; observe that $N^{1}(\Gamma) \subset C^{1}(\Gamma, \mathbb{R})$ is isometric to $\mathbb{Z}^{m} \subset \mathbb{R}^{m}$, where $m$ is the number of edges of $\Gamma$. Dually, if $\Gamma$ is a wedge of loops, then $\operatorname{Im}(d)=\{0\}$ and the lattice $N^{1}(\Gamma)$ is trivial; observe that $\Lambda^{1}(\Gamma) \subset C^{1}(\Gamma, \mathbb{R})$ is again isometric to $\mathbb{Z}^{m} \subset \mathbb{R}^{m}$ where $m$ is the number of loops of $\Gamma$. Whenever appropriate, the reader may assume from now on that the graphs which appear below are neither trees nor wedges of loops.

Let us recall some terminology on graphs (see e.g. [VLW]).

An oriented circuit in a graph $\Gamma$ is an oriented simple closed curve. Each oriented circuit $c$ in $\Gamma$ determines a cochain $g_{c}: \mathbb{E} \rightarrow \mathbb{Z}$ defined by $g_{c}(e)=1$ (resp. $g_{c}(e)=-1$ ) if the oriented edge $e$ appears in $c$ (resp. if $\bar{e}$ appears in $c$ ) and $g_{c}(e)=0$ otherwise; this $g_{c}$ is clearly in $\Lambda^{1}(\Gamma)$.

The girth of $\Gamma$ is the smallest length of a circuit in $\Gamma$.

An oriented cut $a$ in $\Gamma$ is a non empty subset of $\mathbb{E}$ of the following form: there is a partition $V=V_{+}^{a} \sqcup V_{-}^{a}$ of the vertex set $V$ in two non empty subsets, and $a$ is the set of edges with heads in $V_{+}^{a}$ and tails in $V_{-}^{a}$. To each such $a$ corresponds a cochain $g_{a}: \mathbb{E} \rightarrow \mathbb{Z}$ defined by $g_{a}(e)=1$ (resp. $\left.g_{a}(e)=-1\right)$ if the oriented edge $e$ appears in $a$ (resp. if $\bar{e}$ appears in $a$ ) and $g_{a}(e)=0$ otherwise. Denoting by $\chi_{a} \in C^{0}(\Gamma, \mathbb{Z})$ the characteristic function of $V_{+}^{a}$, one has $g_{a}=d\left(\chi_{a}\right) \in N^{1}(\Gamma)$.

If moreover the subgraphs induced by $V_{+}^{a}$ and $V_{-}^{a}$ in $\Gamma$ are connected, we say that $a$ is an oriented bond.

The edge connectivity of $\Gamma$ is the minimal number of edges in a bond (or in a cut) of $\Gamma$ (see [Bol, Chap. 1]).

The complexity of a connected graph is the number of its spanning trees.

The following results are proved in Section 4 below.

Proposition 1. - Let $\Gamma$ be a connected graph.

(i) The lattice $\Lambda^{1}(\Gamma)$ is even if and only if the graph $\Gamma$ is bipartite.

(ii) The minimal norm of $\Lambda^{1}(\Gamma)$ is equal to the girth of $\Gamma$.

(iii) The determinant of $\Lambda^{1}(\Gamma)$ is equal to the complexity of $\Gamma$.

Proposition 2. - Let $\Gamma$ be a connected graph.

(i) The lattice $N^{1}(\Gamma)$ is even if and only if the graph $\Gamma$ is Eulerian (namely if and only if all vertices in $\Gamma$ are of even degrees).

(ii) The minimal norm of $N^{1}(\Gamma)$ is equal to the edge connectivity of $\Gamma$. 
(iii) The determinant of $N^{1}(\Gamma)$ is equal to the complexity of $\Gamma$.

About Part (iii) of Proposition 1, it is classical that the complexity $\kappa(\Gamma)$ of $\Gamma$ is equal to

$$
\frac{1}{|V|} \operatorname{det}^{\prime}\left(\Delta_{0}\right)
$$

where $\operatorname{det}^{\prime}$ indicates the product of the non zero eigenvalues (see Cor. 6.5 in $[\mathrm{Bi1}])$. One has also

$$
\kappa(\Gamma)=\frac{1}{|V|} \operatorname{det}^{\prime}\left(\Delta_{1}\right)
$$

because $d^{*} d$ and $d d^{*}$ have the same non zero eigenvalues (see e.g. [Dix, App. B 26]). But Part (iii) says slightly more, namely that this number $\kappa(\Gamma)$ is the order of the finite abelian group $\left(\Lambda^{1}(\Gamma)\right)^{\sharp} / \Lambda^{1}(\Gamma)$ attached to the graph $\Gamma$ (more on this group in Section 3 below). Thus $\kappa(\Gamma)$ has two natural factorizations. One has firstly

$$
\kappa(\Gamma)=\frac{1}{n} \alpha_{2} \cdots \alpha_{n}
$$

where $\alpha_{2} \leq \cdots \leq \alpha_{n}$ are the non zero eigenvalues of $\Delta_{0}=d^{*} d$ (or equivalently of $\left.\Delta_{1}=d d^{*}\right)$. The group $\left(\Lambda^{1}(\Gamma)\right)^{\sharp} / \Lambda^{1}(\Gamma)$ is canonically isomorphic to a direct product of cyclic groups of orders $n_{1}, \ldots, n_{p}$, where $n_{j}$ divides $n_{j+1}$ for all $j$ in $\{1, \ldots, p-1\}$ (the $n_{j}$ 's are the invariant factors of the finite abelian group). It follows that one has secondly

$$
\kappa(\Gamma)=n_{1} \cdots n_{p}
$$

This factorization is the "canonical factorization" of Berman [Ber], and examples show that it may be quite distinct from the previous factorization (see Section 9 below). Part (iii) of Proposition 1 appears in a different setting as Theorem 3 of [Bi2].

As for any CW-complex (see Remark 4 of Section 9), the first integral cohomology group $H^{1}(\Gamma, \mathbb{Z})$ is torsion free, for example because of the canonical isomorphism $H^{1}(\Gamma, \mathbb{Z}) \approx \operatorname{Hom}\left(\pi_{1}(\Gamma), \mathbb{Z}\right)$. As for any compact CW-complex, there exists a natural embedding $H^{1}(\Gamma, \mathbb{Z}) \hookrightarrow H^{1}(\Gamma, \mathbb{R})$ with cocompact image; this follows for example from the universal coefficient theorem for cohomology. (See e.g. Thm. 12.15 in [Rot]; for graphs, there are of course elementary ad hoc arguments.) For graphs, from the decomposition

$$
C^{1}(\Gamma, \mathbb{R})=\operatorname{Ker}\left(\Delta_{1}\right) \oplus \operatorname{Im}(d),
$$

TOME $125-1997-\mathrm{N}^{\circ} 2$ 
one may identify the real cohomology group $H^{1}(\Gamma, \mathbb{R})$ with the Euclidean space $\operatorname{Ker}\left(\Delta_{1}\right)$ of harmonic 1 -forms, so that $H^{1}(\Gamma, \mathbb{Z})$ is naturally a lattice in $H^{1}(\Gamma, \mathbb{R}) \approx \operatorname{Ker}\left(\Delta_{1}\right)$.

Recall from the end of Section 1 that any lattice $\Lambda$ has a Witt class $w(\Lambda)$ in the group $W(\mathbb{Q} / \mathbb{Z})$.

Proposition 3. - Let $\Gamma$ be a connected graph.

(i) The lattices $\Lambda^{1}(\Gamma)$ and $N^{1}(\Gamma)$ have isomorphic determinant groups.

(ii) One has $w\left(\Lambda^{1}(\Gamma)\right)+w\left(N^{1}(\Gamma)\right)=0$.

(iii) In the space $\operatorname{Ker}\left(\Delta_{1}\right)$, the lattices $\Lambda^{1}(\Gamma)$ and $H^{1}(\Gamma, \mathbb{Z})$ are dual to each other.

We do not know any interesting statement for $N^{1}(\Gamma)$ corresponding to Claim (iii) for $\Lambda^{1}(\Gamma)$.

A cut-vertex in a connected graph $\Gamma$ is a vertex $v$ such that the graph obtained from $\Gamma$ by deleting $v$ and its incident edges has several connected components. A connected graph without cut-vertex is said to be nonseparable (another term for "nonseparable" is 2-connected). The blocks of a connected graph are its maximal nonseparable connected subgraphs. For example, the blocks of a tree with more than one vertex are graphs with two vertices connected by one edge. If a connected graph $\Gamma$ is separable, both $\Lambda^{1}(\Gamma)$ and $N^{1}(\Gamma)$ are orthogonal sums of the contributions of the blocks of $\Gamma$. More generally, one has

Proposition 4. - Let $\Gamma$ be a connected graph which has no loop and no vertex of degree one. The following are equivalent.

(i) The graph $\Gamma$ is nonseparable,

(ii) the lattice $\Lambda^{1}(\Gamma)$ is indecomposable,

(iii) the lattice $N^{1}(\Gamma)$ is indecomposable,

(iv) the polygon matroid of $\Gamma$ is connected.

(For connectedness of matroids, see e.g. Chap. 4 in [Oxl].) About (iv), one should distinguish carefully the polygon matroid $\mathcal{M}(\Gamma)$ and the lattice $\Lambda^{1}(\Gamma)$ of a graph $\Gamma$. Indeed, elements of $\mathcal{M}(\Gamma)$ are given as subsets of the set $E$ of all edges, while the structure of $\Lambda^{1}(\Gamma)$ is defined without its actual embedding in the space $C^{1}(\Gamma, \mathbb{Z})$ of all cochains. For example, if $\Gamma_{1}, \Gamma_{2}$ are two trees with unequal numbers of edges, $\mathcal{M}\left(\Gamma_{1}\right), \mathcal{M}\left(\Gamma_{2}\right)$ are not isomorphic, but $\Lambda^{1}\left(\Gamma_{1}\right) \approx \Lambda^{1}\left(\Gamma_{2}\right) \approx\{0\}$.

Let $\Gamma$ be a connected nonseparable graph. Suppose that $\Gamma$ is obtained from the disjoint graphs $\Gamma_{1}, \Gamma_{2}$ by identifying the vertices $u_{1}$ of $\Gamma_{1}$ and $u_{2}$ of $\Gamma_{2}$ as the vertex $u$ of $\Gamma$ and the vertices $v_{1}$ of $\Gamma_{1}$ and $v_{2}$ of $\Gamma_{2}$ as the vertex $v$ of $\Gamma$. Then $\Gamma^{\prime}$ is a twisting of $\Gamma$ about $\{u, v\}$ if it is 
obtained from $\Gamma_{1}$ and $\Gamma_{2}$ by identifying $u_{1}$ with $v_{2}$ and $v_{1}$ with $u_{2}$. Two connected nonseparable graphs are said to be 2-isomorphic if they can be transformed into isomorphic graphs by a sequence of twistings. Notice that 3-connected 2-isomorphic graphs are isomorphic.

As the graphic matroids (see [Oxl, p. 148]), the lattices $\Lambda^{1}(\Gamma)$ and $N^{1}(\Gamma)$ are invariant under 2-isomorphisms of graphs. More precisely, one has

Proposition 5. - If two graphs $\Gamma, \Gamma^{\prime}$ are 2-isomorphic, one has isomorphisms of lattices

$$
\Lambda^{1}(\Gamma) \approx \Lambda^{1}\left(\Gamma^{\prime}\right) \quad \text { and } \quad N^{1}(\Gamma) \approx N^{1}\left(\Gamma^{\prime}\right)
$$

Let $\Lambda$ be a lattice in a Euclidean space $\mathcal{H}$. The Voronoi polyhedron of $\Lambda$ is the fundamental domain

$$
\text { Vor }_{\Lambda}=\{x \in \mathcal{H} \mid\langle x, x\rangle \leq\langle x-\lambda, x-\lambda\rangle \text { for all } \lambda \in \Lambda \backslash\{0\}\}
$$

for the action of $\Lambda$ on $\mathcal{H}$ by translations. It is a compact convex polytope.

A vector $\lambda \in \Lambda$ is said to be relevant if it corresponds to a codimensionone-face of the Voronoi polyhedron. Otherwise said, if $\mathcal{H}_{\lambda}$ denotes the hyperplan of equation $\langle x, x\rangle=\langle x-\lambda, x-\lambda\rangle$ in $\mathcal{H}$, the vector $\lambda$ is relevant if $\lambda \neq 0$ and if $\operatorname{Vor}_{\Lambda} \cap \mathcal{H}_{\lambda}$ contains a non empty open subset of $\mathcal{H}_{\lambda}$.

Proposition 6. - Let $\Gamma$ be a connected graph.

(i) There is a natural bijection between oriented circuits of $\Gamma$ and faces of codimension 1 of the Voronoi polyhedron of $\Lambda^{1}(\Gamma)$; more precisely, a vector $\lambda \in \Lambda^{1}(\Gamma)$ is relevant if and only if it is of the form $g_{c}$ for some oriented circuit $c$ of $\Gamma$.

(ii) There is a natural bijection between oriented bonds of $\Gamma$ and faces of codimension 1 of the Voronoi polyhedron of $N^{1}(\Gamma)$; more precisely, a vector $\nu \in N^{1}(\Gamma)$ is relevant if and only if it is of the form $g_{b}$ for some oriented bond $b$ of $\Gamma$.

(For the notations $g_{c}, g_{b}$ see the beginning of Section 2.) Similar characterization for the faces of Voronoi polyhedra appear in Prop. 5.2 of [OdS]; our proof below is simpler and more direct.

It would be interesting to understand when two oriented circuits $c_{1}, c_{2}$ in $\Gamma$ correspond to faces $F_{1}, F_{2}$ of the Voronoi polyhedron for $\Lambda^{1}(\Gamma)$ such that $F_{1} \cap F_{2}$ is of codimension 2 in $\operatorname{Ker}\left(\Delta_{1}\right)$. More ambitiously, one would like to understand the combinatorics of this polyhedron in terms of oriented circuits in the graph. (And similarly for oriented bonds and $N^{1}(\Gamma)$.) 


\section{The Jacobian of a graph}

Graphs give rise to a toy-kit which refers to classical achievements of last century analysis. Let us first recall the following notions, for which one out of many good references is Narasimhan's lecture course [Nar].

Let $X$ be a compact Riemann surface. Denote by $H^{0}(X, \Omega)$ the space of holomorphic 1-forms on $X$ (which is finite dimensional), by $H^{0}(X, \Omega)^{*}$ the dual space and by $H_{1}(X, \mathbb{Z})$ the first integral homology group of $X$. There is a positive definite sesquilinear form

$$
\left\{\begin{array}{l}
H^{0}(X, \Omega) \times H^{0}(X, \Omega) \longrightarrow \mathbb{C}, \\
\left(\omega_{1}, \omega_{2}\right) \longmapsto \int_{X} \omega_{1} \wedge \bar{\omega}_{2}
\end{array}\right.
$$

and a corresponding sesquilinear form on the dual space $H^{0}(X, \Omega)^{*}$. There is also a natural mapping

$$
H_{1}(X, \mathbb{Z}) \longrightarrow H^{0}(X, \Omega)^{*}
$$

which applies the homology class of a loop $\gamma:[0,1] \rightarrow X$ to the linear form $[\omega] \mapsto \int_{\gamma} \omega$ on $H^{0}(X, \Omega)$. This mapping is an isomorphism of $H_{1}(X, \mathbb{Z})$ onto a lattice in $H^{0}(X, \Omega)^{*}$ and the quotient

$$
\mathcal{J}(X)=H^{0}(X, \Omega)^{*} / H_{1}(X, \mathbb{Z})
$$

is the Jacobian of $X$.

Given a base point $x_{0} \in X$, the Abel-Jacobi map

$$
A: X \longrightarrow \mathcal{J}(X)
$$

applies a point $x \in X$ to the class in $\mathcal{J}(X)$ of the linear form $\omega \mapsto \int_{\gamma} \omega$ on $H^{0}(X, \Omega)$, where $\gamma$ is some path from $x_{0}$ to $x$. For each integer $N \geq 1$, let $S^{N}(X)$ denote the quotient of $X^{N}$ by the obvious action of the symmetric group on $N$ letters. One has also a map

$$
A^{N}: S^{N}(X) \longrightarrow \mathcal{J}(X)
$$

which applies the class of $\left(x_{1}, \ldots, x_{N}\right)$ to $\sum_{j=1}^{N} A\left(x_{j}\right)$.

BULLETIN DE LA SOCIÉTÉ MATHÉMATIQUE DE FRANCE 
Let $\operatorname{Div}(X)$ be the group of divisors of $X$, which is the free abelian group on $X$. Let $\operatorname{Div}^{0}(X)$ denote the subgroup of divisors of degree zero, i.e. of divisors of the form

$$
\sum_{x \in X} n_{x} x \text { with } \sum_{x \in X} n_{x}=0 .
$$

The group $P(X)$ of principal divisors (i.e. of divisors of meromorphic functions on $X$ ) is a subgroup of $\operatorname{Div}^{0}(X)$ and the quotient

$$
\operatorname{Pic}(X)=\operatorname{Div}^{0}(X) / P(X)
$$

is the Picard group of $X$.

Let us recall the following classical results, where $g=\operatorname{dim}_{\mathbb{C}} H^{0}(X, \Omega)$ denotes the genus of $X$; we assume $g \geq 1$.

1) The map $A^{N}: S^{N}(X) \rightarrow \mathcal{J}(X)$ is holomorphic for all $N \geq 1$.

2) If $g=1$, the map $A: X \rightarrow \mathcal{J}(X)$ is an isomorphism.

3) The Abel-Jacobi map induces a group homomorphism $\operatorname{Pic}(X) \rightarrow$ $\mathcal{J}(X)$ which is an isomorphism.

4) The map $A: X \rightarrow \mathcal{J}(X)$ is an embedding.

5) The map $A^{g}: S^{g}(X) \rightarrow \mathcal{J}(X)$ is birational and onto (Abel's theorem).

Moreover, $\mathcal{J}(X)$ determines $X$ in the appropriate sense (this is Torelli's Theorem; see [Nar]).

Let $\Gamma$ be a connected graph. As before (see before Proposition 3 ) we identify the cohomology group $H^{1}(\Gamma, \mathbb{R})$ to $\operatorname{Ker}\left(\Delta_{1}\right)$, so that one has an orthogonal projection $\pi: C^{1}(\Gamma, \mathbb{R}) \rightarrow H^{1}(\Gamma, \mathbb{R})$. We denote by

$$
\widetilde{A}: C^{1}(\Gamma, \mathbb{Z}) \longrightarrow\left(\Lambda^{1}(\Gamma)\right)^{\sharp}
$$

the restriction of $\pi$ to the group of integral cochains (see Lemma 1 of Section 4). For $e \in \mathbb{E}$, let $\delta_{e} \in C^{1}(\Gamma, \mathbb{Z})$ be defined by

$$
\delta_{e}(e)=1, \quad \delta_{e}(\bar{e})=-1 \text { and } \delta_{e}\left(e^{\prime}\right)=0 \text { for } e^{\prime} \in \mathbb{E} \backslash\{e, \bar{e}\}
$$

observe that $\widetilde{A}\left(\delta_{e}\right)=0$ if and only if $e$ is an isthmus (i.e. is contained in no cycle), that $\widetilde{A}\left(\delta_{e}\right)$ is of length one if and only if $e$ is a loop, and consequently that

$$
0<\left\langle\widetilde{A}\left(\delta_{e}\right), \widetilde{A}\left(\delta_{e}\right)\right\rangle<1
$$

in all other cases.

TOME $125-1997-\mathrm{N}^{\circ} 2$ 
Choose a base point $v_{0} \in V$. For each $v \in V$ and for each oriented path $\gamma$ from $v_{0}$ to $v$, one has a corresponding element

$$
\widetilde{A}(\gamma)=\sum_{e \in \gamma} \widetilde{A}\left(\delta_{e}\right) \in\left(\Lambda^{1}(\Gamma)\right)^{\sharp} .
$$

If $\gamma$ is a closed oriented path from $v_{0}$ to $v_{0}$, one has clearly $\widetilde{A}(\gamma) \in \Lambda^{1}(\Gamma)$. It follows that there is a well defined map

$$
A_{v_{0}}: V \longrightarrow\left(\Lambda^{1}(\Gamma)\right)^{\sharp} / \Lambda^{1}(\Gamma) .
$$

Definition. - The Jacobian of a finite connected graph $\Gamma$ is the determinant group

$$
\mathcal{J}(\Gamma)=\left(\Lambda^{1}(\Gamma)\right)^{\sharp} / \Lambda^{1}(\Gamma)
$$

of its lattice of integral flows. Given $v_{0} \in V$, the corresponding Abel-Jacobi map is the map $A_{v_{0}}: V \rightarrow \mathcal{J}(\Gamma)$ defined above.

The map $\widetilde{A}: C^{1}(\Gamma, \mathbb{Z}) \rightarrow\left(\Lambda^{1}(\Gamma)\right)^{\sharp}$ is onto (by Lemma 1 ), so that $\left(\Lambda^{1}(\Gamma)\right)^{\sharp}$ is generated by elements of the form $\widetilde{A}(\gamma)$. It follows that the image of $A_{v_{0}}$ is a set of generators for $\mathcal{J}(\Gamma)$.

Let $\operatorname{Div}(\Gamma)$ denote the free abelian group on the vertices of $\Gamma$. (This is, viewed differently, the group $C^{0}(\Gamma, \mathbb{Z})$ of integral cochains.) Let

$$
\operatorname{Div}^{0}(\Gamma)=\left\{\sum_{v \in V} n_{v} v \in \operatorname{Div}(\Gamma) \mid \sum_{v \in V} n_{v}=0\right\}
$$

be the group of divisors of degree zero. (This is, viewed differently, the group $M^{0}(\Gamma)$ of Section 1.) Given any $v_{0} \in V$, one may see $\operatorname{Div}^{0}(\Gamma)$ as the free abelian group on the generators $v-v_{0}$ (for $v \in V$ and $v \neq v_{0}$ ). The restriction of $d^{*}$ to integral cochains is a group homomorphism

$$
d^{*}: C^{1}(\Gamma, \mathbb{Z}) \longrightarrow \operatorname{Div}^{0}(\Gamma)
$$

which is onto because, when $e$ describes $\mathbb{E}$, the images $d^{*}\left(\delta_{e}\right)=\delta_{e_{+}}-\delta_{e_{-}}$ generate $\operatorname{Div}^{0}(\Gamma)$. Let

$$
P(\Gamma)=d^{*}\left(N^{1}(\Gamma)\right)
$$

be the subgroup of principal divisors of $\Gamma$.

Definition. - The Picard group of a finite connected graph $\Gamma$ is the quotient

$$
\operatorname{Pic}(\Gamma)=\operatorname{Div}^{0}(\Gamma) / P(\Gamma)
$$

of the group of divisors of degree zero by the group of principal divisors. Given $v_{0} \in V$, we denote by $P_{v_{0}}: V \rightarrow \operatorname{Pic}(\Gamma)$ the map which assigns to $v \in V$ the class of $v-v_{0}$ in $\operatorname{Pic}(\Gamma)$.

Observe that the image of $P_{v_{0}}$ generates the group $\operatorname{Pic}(\Gamma)$. 
Definition. - A harmonic map from a graph $\Gamma=(V, \mathbb{E})$ to an abelian group $G$ (written additively here) is a map $h: V \rightarrow G$ such that

$$
\operatorname{deg}(v) h(v)=\sum_{\substack{w \in V \\ w \sim v}} h(w) \text { for all } v \in V,
$$

where $\operatorname{deg}(v)$ is the number of non oriented edges incident to $v$ in $\Gamma$ and where $v \sim w$ indicates that the sum is taken over all edges $e \in \mathbb{E}$ for which $e_{-}=w$ and $e_{+}=v$. (Our "harmonic maps" are the "balanced maps" of Berman [Ber].)

OBSERVATION. - Let $h: V \rightarrow G$ be a harmonic map as above; assume that $h\left(v_{0}\right)=0$ for some $v_{0} \in V$. Then there exists a unique group homomorphism $\tilde{h}: \operatorname{Pic}(\Gamma) \rightarrow G$ such that $h=\tilde{h} \circ P_{v_{0}}$.

Proof. - $\operatorname{As} \operatorname{Div}^{0}(\Gamma)$ is the free abelian group on $V \backslash\left\{v_{0}\right\}$, any map $h: V \rightarrow G$ such that $h\left(v_{0}\right)=0$ provides naturally a group homomorphism $\hat{h}: \operatorname{Div}^{0}(\Gamma) \rightarrow G$ such that $\hat{h}\left(v-v_{0}\right)=h(v)$ for all $v \in V$.

As $N^{1}(\Gamma)$ is generated by the $d\left(\delta_{v}\right)$ 's for $v \in V$, the group $P(\Gamma)$ is generated by the $d^{*} d\left(\delta_{v}\right)$ 's for $v \in V$, namely by the divisors

$$
\operatorname{deg}(v) v-\sum_{\substack{w \in V \\ w \sim v}} w
$$

Thus $\hat{h}$ factors through $\operatorname{Pic}(\Gamma)$ if and only if $h$ is harmonic.

When $h$ is harmonic, the unicity of $\tilde{h}$ follows from the fact that the image of $P_{v_{0}}$ generates $\operatorname{Pic}(\Gamma)$.

In analogy with Results (1) to (4) recalled above for Riemann surfaces, we offer the following.

Proposition 7. - Let $\Gamma$ be a connected graph and let $v_{0}$ be a vertex of $\Gamma$. The notations being as above, one has the following:

(i) the map $A_{v_{0}}: V \rightarrow \mathcal{J}(\Gamma)$ is harmonic and its image generates $\mathcal{J}(\Gamma)$;

(ii) if $\Gamma$ is a polygon, then $A_{v_{0}}$ is a bijection;

(iii) the group homomorphism $\operatorname{Pic}(\Gamma) \rightarrow \mathcal{J}(\Gamma)$ induced by $A_{v_{0}}$ is an isomorphism and applies $P_{v_{0}}(v)$ onto $A_{v_{0}}(v)$ for all $v \in V$.

If moreover the graph $\Gamma$ is nonseparable, and not the link-graph made of one edge connecting two distinct vertices, then

(iv) the map $A_{v_{0}}: V \rightarrow \mathcal{J}(\Gamma)$ is injective.

Let $\widetilde{S}$ be the subset of $\left(\Lambda^{1}(\Gamma)\right)^{\sharp}$ of elements of the form $\widetilde{A}\left(\delta_{e}\right), e \in \mathbb{E}$, and denote by $S$ the canonical projection of $\widetilde{S}$ in $\mathcal{J}(\Gamma)$. Then $S$ is a

$$
\text { TOME } 125-1997-\mathrm{N}^{\circ} 2
$$


symmetric set of generators of $\mathcal{J}(\Gamma)$, because $\left(\delta_{e}\right)_{e \in \mathbb{E}}$ contains a basis of $C^{1}(\Gamma, \mathbb{Z})$. It is the corresponding Cayley graph

$$
\operatorname{Cay}(\mathcal{J}(\Gamma), S)
$$

that should probably be called the Jacobian of $\Gamma$, rather than the group $\mathcal{J}(\Gamma)$ itself. Observe that, for each $v_{0} \in V$ and under the hypothesis of (iv) in Proposition 7, the map $A_{v_{0}}$ may be viewed as an isomorphism of $\Gamma$ onto a subgraph of $\operatorname{Cay}(\mathcal{J}(\Gamma), S)$. It is an open problem for us to obtain a good analogue of Abel's theorem in our setting (see Result (5) recalled above); here are more precise formulations of this problem. (The radius of a Cayley graph $\operatorname{Cay}(G, S)$ is the largest combinatorial distance between the vertex 1 and another vertex $g$ of the graph.)

(v) How do the radius and diameter of $\operatorname{Cay}(\mathcal{J}(\Gamma), S)$ depend on the geometry of $\Gamma$ ?

$\left(\mathrm{v}^{\prime}\right)$ For each vertex $v_{0} \in V$ and for each integer $N$, one has a natural map

$$
A_{v_{0}}^{N}: S^{N}(V) \longrightarrow \mathcal{J}(\Gamma)
$$

Let $g_{\mathrm{Jac}}\left(\Gamma, v_{0}\right)$ denote the smallest integer $N$ for which $A_{v_{0}}^{N}$ is onto. How does this "Jacobian genus" of $\Gamma$ depend on the graph, and possibly on the base point $v_{0}$ ?

A naive analogue of Torelli's Theorem does not hold, because there are pairs of non-isomorphic graphs with isomorphic Jacobians. Examples are given by planar graphs and their duals, as it follows from our last proposition.

Proposition 8. - Let $\Gamma$ be a planar graph and $\Gamma^{*}$ a planar dual of $\Gamma$. Then $\Lambda^{1}(\Gamma)$ is isomorphic to $N^{1}\left(\Gamma^{*}\right)$ and $N^{1}(\Gamma)$ is isomorphic to $\Lambda^{1}\left(\Gamma^{*}\right)$. In particular the Jacobians $\mathcal{J}(\Gamma)$ and $\mathcal{J}\left(\Gamma^{*}\right)$ are isomorphic abelian groups. Moreover the Witt classes satisfy

$$
w\left(\Lambda^{1}(\Gamma)\right)+w\left(\Lambda^{1}\left(\Gamma^{*}\right)\right)=0 \in W(\mathbb{Q} / \mathbb{Z}) .
$$

\section{A lemma on lattices and proof of Proposition 3}

Claims (i)-(iii) of the following Lemma essentially repeat Theorem 1 in Chapter 4 of $[\mathrm{CoS}]$, for which we give a proof.

LemMA 1. - Let $\mathcal{H}$ be a Euclidean space and let $\mathcal{F}, \mathcal{G}$ be two orthogonal subspaces, not reduced to $\{0\}$, such that $\mathcal{H}=\mathcal{F} \oplus \mathcal{G}$. Let $\pi_{\mathcal{F}}$ (resp. $\left.\pi_{\mathcal{G}}\right)$ denote the orthogonal projection of $\mathcal{H}$ onto $\mathcal{F}$ (resp. onto $\mathcal{G})$. We assume the situation defined over $\mathbb{Q}$ in the following sense: $\mathcal{H}$ is given together with a $\mathbb{Q}$-subspace $\mathcal{H}_{\mathbb{Q}}$ such that

$$
\operatorname{dim}_{\mathbb{Q}}\left(\mathcal{H}_{\mathbb{Q}}\right)=\operatorname{dim}_{\mathbb{R}}(\mathcal{H}), \quad \mathcal{H}_{\mathbb{Q}}=\left(\mathcal{H}_{\mathbb{Q}} \cap \mathcal{F}\right) \oplus\left(\mathcal{H}_{\mathbb{Q}} \cap \mathcal{G}\right)
$$


and $\langle x, y\rangle \in \mathbb{Q}$ for all $x, y \in \mathcal{H}_{\mathbb{Q}}$. Let $\Lambda$ be a unimodular integral lattice in $\mathcal{H}$ such that $\Lambda \subset \mathcal{H}_{\mathbb{Q}}$. Set $M=\Lambda \cap \mathcal{F}$ and $N=\Lambda \cap \mathcal{G}$. Then

(i) $M$ is a lattice in $\mathcal{F}$ and $N$ is a lattice in $\mathcal{G}$,

(ii) $M^{\sharp}=\pi_{\mathcal{F}}(\Lambda)$ and $N^{\sharp}=\pi_{\mathcal{G}}(\Lambda)$,

(iii) the lattices $M \subset \mathcal{F}$ and $N \subset \mathcal{G}$ have isomorphic determinant groups,

(iv) the Witt classes of $M$ and $N$ satisfy $w(M)+w(N)=0$ in the Witt group $W(\mathbb{Q} / \mathbb{Z})$.

Proof.

Claim (i). This holds because $\mathcal{H}_{\mathbb{Q}}$ coincides with the subset of $\mathcal{H}$ of those vectors $x$ for which some integral multiple $n x$ is in $\Lambda$.

Claim (ii). Let $r$ denote the dimension of $\mathcal{F}$. Choose a $\mathbb{Z}$-basis $\left\{b_{1}, \ldots, b_{r}\right\}$ of $M$. As $\Lambda / M$ is torsion-free, one may find a $\mathbb{Z}$-basis

$$
\left\{b_{1}, \ldots, b_{r}, b_{r+1}, \ldots, b_{m}\right\}
$$

of $\Lambda$ (see [Bou, VII, p. 18, cor.]). Let $\left\{b_{1}^{\prime}, \ldots, b_{m}^{\prime}\right\}$ denote the dual basis defined by $\left\langle b_{j}^{\prime}, b_{k}\right\rangle=\delta_{j, k}$ for $j, k \in\{1, \ldots, m\}$; it is again a $\mathbb{Z}$-basis of $\Lambda$ because $\Lambda$ is unimodular. As $\pi_{\mathcal{F}}\left(b_{j}^{\prime}\right)=0$ for $j>r$, the set $\left\{\pi_{\mathcal{F}}\left(b_{1}^{\prime}\right), \ldots, \pi_{\mathcal{F}}\left(b_{r}^{\prime}\right)\right\}$ is a $\mathbb{Z}$-basis of $\pi_{\mathcal{F}}(\Lambda)$. Any $x \in \mathcal{F}$ is of the form

$$
\sum_{j=1}^{r} x_{j} \pi_{\mathcal{F}}\left(b_{j}^{\prime}\right)
$$

where $x_{1}, \ldots, x_{r} \in \mathbb{R}$; and $\langle x, M\rangle \in \mathbb{Z}$ if and only if $\left\langle x, b_{j}\right\rangle \in \mathbb{Z}$ for $j \in\{1, \ldots, r\}$, namely if and only if $x_{1}, \ldots, x_{r} \in \mathbb{Z}$. It follows that $M^{\sharp}$ and $\pi_{\mathcal{F}}(\Lambda)$ coincide. Similarly $N^{\sharp}=\pi_{\mathcal{G}}(\Lambda)$.

Claim (iii). Consider now the restriction $\Lambda \rightarrow M^{\sharp}$ of $\pi_{\mathcal{F}}$. It factorizes firstly as a map $\Lambda / N \rightarrow M^{\sharp}$, and secondly as a map

$$
\varphi_{M}: \Lambda /(M \oplus N) \longrightarrow M^{\sharp} / M
$$

which is onto by (ii). Choose $\lambda \in \Lambda$ and denote by $[\lambda]$ its class modulo $M \oplus N$. Assume that $\varphi_{M}([\lambda])=0$, namely that $\pi_{\mathcal{F}}(\lambda) \in M$; as $\lambda-\pi_{\mathcal{F}}(\lambda)$ is in $\Lambda \cap \operatorname{Ker}\left(\pi_{\mathcal{F}}\right)$, one has $\lambda-\pi_{\mathcal{F}}(\lambda) \in N$ and consequently $\lambda \in M \oplus N$. It follows that $\varphi_{M}$ is injective, namely that $\varphi_{M}$ is an isomorphism. As one has similarly an isomorphism

$$
\varphi_{N}: \Lambda /(M \oplus N) \longrightarrow N^{\sharp} / N
$$

this ends the proof of Claim (iii).

$$
\text { TOME } 125-1997-\mathrm{N}^{\circ} 2
$$


Claim (iv). Let $\varphi: M^{\sharp} / M \rightarrow N^{\sharp} / N$ be the isomorphism of (iii). For each pair $\underline{m}_{1}, \underline{m}_{2} \in M^{\sharp} / M$, choose a pair of vectors $\lambda_{1}, \lambda_{2} \in \Lambda$ such that $\pi_{\mathcal{F}}\left(\lambda_{1}\right), \pi_{\mathcal{F}}\left(\lambda_{2}\right)$ are respectively representants of $\underline{m}_{1}$ and $\underline{m}_{2}$. Then $\pi_{\mathcal{G}}\left(\lambda_{1}\right)$, $\pi_{\mathcal{G}}\left(\lambda_{2}\right)$ are representants of $\varphi\left(\underline{m}_{1}\right), \varphi\left(\underline{m}_{2}\right) \in N^{\sharp} / N$. As

$$
\left\langle\pi_{\mathcal{F}}\left(\lambda_{1}\right), \pi_{\mathcal{F}}\left(\lambda_{2}\right)\right\rangle+\left\langle\pi_{\mathcal{G}}\left(\lambda_{1}\right), \pi_{\mathcal{G}}\left(\lambda_{2}\right)\right\rangle=\left\langle\lambda_{1}, \lambda_{2}\right\rangle \in \mathbb{Z},
$$

one has

$$
\left\langle\pi_{\mathcal{F}}\left(\lambda_{1}\right), \pi_{\mathcal{F}}\left(\lambda_{2}\right)\right\rangle \equiv-\left\langle\pi_{\mathcal{G}}\left(\lambda_{1}\right), \pi_{\mathcal{G}}\left(\lambda_{2}\right)\right\rangle \bmod \mathbb{Z} .
$$

Thus, with the notations of Section $1,\left(M^{\sharp} / M, b_{M}\right) \approx\left(N^{\sharp} / N,-b_{N}\right)$, namely $w(M)+w(N)=0 \in W(\mathbb{Q} / \mathbb{Z})$.

Proof of Proposition 3. - The first two claims of Proposition 3 are particular cases of the claims of Lemma 1.

For Claim (iii) of the Proposition, observe that the cohomology space $H^{1}(\Gamma, \mathbb{R})=C^{1}(\Gamma, \mathbb{R}) / \operatorname{Im}(d)$ may be viewed as the image of the first projection with respect to the orthogonal decomposition

$$
C^{1}(\Gamma, \mathbb{R})=\operatorname{Ker}\left(\Delta_{1}\right) \oplus \operatorname{Im}(d) .
$$

Therefore the integral cohomology $H^{1}(\Gamma, \mathbb{Z})$, which is a discrete subgroup of $H^{1}(\Gamma, \mathbb{R})$, can be viewed as the orthogonal projection of $C^{1}(\Gamma, \mathbb{Z})$ on $\operatorname{Ker}\left(\Delta_{1}\right)$. Claim (iii) of Proposition 3 is consequently a particular case of Lemma 1 (ii).

\section{Proof of (i) and (iii) in Propositions 1 and 2.}

As in Sections 1 and 2, we consider a connected graph $\Gamma=(V, E)$ and the lattices

$$
\Lambda^{1}(\Gamma) \subset \operatorname{Ker}\left(\Delta_{1}\right), \quad N^{1}(\Gamma) \subset \operatorname{Im}(d) .
$$

Recall from Section 2 that each oriented circuit $c$ in $\Gamma$ defines a 1 -form $g_{c} \in \Lambda^{1}(\Gamma)$ and that each oriented bond $b$ in $\Gamma$ defines a 1-form $g_{b} \in N^{1}(\Gamma)$. Let $T$ be a spanning tree in $\Gamma$; denote by $\mathbb{T}$ the set of oriented edges of $T$. For any edge $e \in \mathbb{E}$ not in $\mathbb{T}$, there is a unique oriented circuit $c(e)$ in $\Gamma$ containing $e$ and edges in $\mathbb{T}$. For any edge $e \in \mathbb{E}$ which is in $\mathbb{T}$, there is a unique oriented bond $b(e)$ containing $e$ and edges not in $\mathbb{T}$.

Let $\mathbb{F}$ be a subset of $\mathbb{E}$ such that $e \in \mathbb{F}$ if and only if $\bar{e} \in \mathbb{F}$. An orientation of $\mathbb{F}$ is a choice of a subset $\mathcal{O}(\mathbb{F})$ of $\mathbb{F}$ which contains exactly one oriented edge from each pair $\{f, \bar{f}\} \subset \mathbb{F}$. Let $\mathcal{O}(\mathbb{E} \backslash \mathbb{T})$ be an orientation of $\mathbb{E} \backslash \mathbb{T}$ and let $\mathcal{O}(\mathbb{T})$ be an orientation of $\mathbb{T}$; they constitute together an orientation of $\mathbb{E}$, also called an orientation of the graph $\Gamma$. 
It is well known that

- $\left(g_{c(e)}\right)_{e \in \mathcal{O}(\mathbb{E} \backslash \mathbb{T})}$ is a basis of $\operatorname{Ker}\left(\Delta_{1}\right)$,

- $\left(g_{b(e)}\right)_{e \in \mathcal{O}(\mathbb{T})}$ is a basis of $\operatorname{Im}(d)$

(see Theorem 5.2 in [Bi1]). More precisely, one has the following.

Lemma 2. - Let $T$ be a spanning tree in $\Gamma$ and let $\mathcal{O}(\mathbb{E}) \subset \mathbb{E}$ be an orientation of $\Gamma$. The notations being as above, one has:

- $\left(g_{c(e)}\right)_{e \in \mathcal{O}(\mathbb{E} \backslash \mathbb{T})}$ is a $\mathbb{Z}$-basis of the lattice $\Lambda^{1}(\Gamma)$ in $\operatorname{Ker}\left(\Delta_{1}\right)$,

- $\left(g_{b(e)}\right)_{e \in \mathcal{O}(\mathbb{T})}$ is a $\mathbb{Z}$-basis of the lattice $N^{1}(\Gamma)$ in $\operatorname{Im}(d)$. Then

Proof.-Choose an orientation $\mathcal{O}(\mathbb{E})$ of $\Gamma$ and let $\lambda=\sum_{i} \lambda(e) \delta_{e} \in \Lambda^{1}(\Gamma)$.

$$
\lambda-\sum_{e \in \mathcal{O}(\mathbb{E} \backslash \mathbb{T})} \lambda(e) g_{c(e)} \in \Lambda^{1}(\Gamma)
$$

may be viewed as an integral harmonic 1-form on $T$ and is consequently zero. Hence $\left(g_{c(e)}\right)_{e \in \mathcal{O}(\mathbb{E} \backslash \mathbb{T})}$ is a $\mathbb{Z}$-basis of $\Lambda^{1}(\Gamma)$.

Let $\nu=\sum_{e \in \mathcal{O}(\mathbb{E})} \nu(e) \delta_{e} \in N^{1}(\Gamma)$. Then

$$
\nu-\sum_{e \in \mathcal{O}(\mathbb{T})} \nu(e) g_{b(e)} \in N^{1}(\Gamma)
$$

may be viewed as a boundary whose restriction to $T$ is zero, and is consequently zero as a boundary of $\Gamma$. Hence $\left(g_{b(e)}\right)_{e \in \mathcal{O}(\mathbb{T})}$ is a $\mathbb{Z}$-basis of $N^{1}(\Gamma)$.

\section{Proof of (i) and (iii) in Propositions 1 and 2.}

The graph $\Gamma$ is bipartite if and only if all its circuits have even lengths. The length of an oriented circuit $c$ of $\Gamma$ is the norm $\left\langle g_{c}, g_{c}\right\rangle$ where $g_{c}$ is as above. Hence Claim (i) of Proposition 1 follows from Lemma 2.

The graph is Eulerian if and only if all its vertices have even degrees. Let $v_{1}, \ldots, v_{n}$ be an enumeration of the vertex set $V$ of $\Gamma$. For each $j \in\{1, \ldots, n\}$, let $\delta_{j} \in C^{0}(\Gamma, \mathbb{R})$ denote the characteristic function of $v_{j}$. The degree of $v_{j}$ is the norm $\left\langle d\left(\delta_{j}\right), d\left(\delta_{j}\right)\right\rangle$. Now $\left(d\left(\delta_{j}\right)\right)_{1 \leq j \leq n-1}$ is a basis of the vector space $\operatorname{Im}(d)$, because $\Gamma$ is connected, and it is also a $\mathbb{Z}$-basis of $N^{1}(\Gamma)$, because

$$
d\left(C^{0}(\Gamma, \mathbb{Z})\right)=C^{1}(\Gamma, \mathbb{Z}) \cap \operatorname{Im}(d) .
$$

Claim (i) of Proposition 2 follows.

TOME $125-1997-\mathrm{N}^{\circ} 2$ 
The determinant of the lattice $N^{1}(\Gamma)$ is the determinant of its Gram matrix $G=\left(\left\langle d\left(\delta_{j}\right), d\left(\delta_{k}\right)\right\rangle\right)_{1 \leq j, k \leq n-1}$, and one has

$$
\left\langle d\left(\delta_{j}\right), d\left(\delta_{k}\right)\right\rangle= \begin{cases}\operatorname{deg}\left(v_{j}\right) & \text { if } k=j, \\ -\sharp\left\{\text { edges between } v_{j} \text { and } v_{k}\right\} & \text { otherwise }\end{cases}
$$

(we agree here that loops do not contribute to degrees). It is a standard result that the determinant of $G$ is precisely the complexity of the graph $\Gamma$ (see Theorem 6.3 in [Bi1]). Lemma 1 shows that the determinant of $N^{1}(\Gamma)$ is equal to that of $\Lambda^{1}(\Gamma)$. Claims (iii) of Propositions 1 and 2 follow.

Claims (ii) in Propositions 1 and 2 could be proved now, but they are also straightforward consequences of Proposition 6, proved in Section 7 below.

\section{Proof of Propositions 4 and 5}

Given a graph $\Gamma$, the circuit graph $\operatorname{Cir}(\Gamma)$ of $\Gamma$ is the following simple graph:

- its vertices are the circuits in $\Gamma$ (a circuit being here an unoriented simple closed curve, possibly a loop);

- two circuits $c_{1}, c_{2}$ of $\Gamma$ are connected by an edge in $\operatorname{Cir}(\Gamma)$ if they have at least one common edge in $\Gamma$.

For example, if $\Gamma$ is a complete graph with 4 vertices, then $\operatorname{Cir}(\Gamma)$ is a complete graph with 7 vertices.

It follows from the definitions that saying that $\operatorname{Cir}(\Gamma)$ is connected is a reformulation of saying that the polygon matroid of $\Gamma$ is connected.

LemMA 3. - Let $\Gamma$ be a connected graph which has no loop and no vertex of degree 1 . Then the following are equivalent:

(i) $\Gamma$ is separable,

(ii) $\operatorname{Cir}(\Gamma)$ is not connected,

(iii) $\Lambda^{1}(\Gamma)$ is decomposable.

Proof.

(i) $\Rightarrow$ (ii) and (iii). Let $\Gamma_{1}, \ldots, \Gamma_{b}$ be the blocks of $\Gamma$, with $b \geq 2$. As $\Gamma$ has no vertex of degree 1 , none of the graphs $\operatorname{Cir}\left(\Gamma_{j}\right)$ is empty, and $\operatorname{Cir}(\Gamma)$ is the disjoint union of these. For the same reason, none of the lattices $\Lambda^{1}\left(\Gamma_{j}\right)$ is reduced to $\{0\}$, and $\Lambda^{1}(\Gamma)$ is the orthogonal sum of these.

(ii) $\Rightarrow$ (i). Let $\mathrm{Cir}_{1}, \ldots, \mathrm{Cir}_{b}$ denote the connected components of $\operatorname{Cir}(\Gamma)$, with $b \geq 2$. For each $j \in\{1, \ldots, b\}$, set

$E_{j}=\left\{e \in E \mid e\right.$ belongs to at least one circuit contributing to $\left.\mathrm{Cir}_{j}\right\}$ 
and let $\Gamma_{j}$ denote the subgraph of $\Gamma$ consisting of edges in $E_{j}$ and their incident vertices. One has $E_{1} \cup \ldots \cup E_{b}=E$ because $\Gamma$ has no vertex of degree 1 , and this is a disjoint union by definition of the $\mathrm{Cir}_{j}$ 's. It follows that $\Gamma_{1}, \ldots, \Gamma_{b}$ are the blocks of $\Gamma$, and $\Gamma$ is separable because $b \geq 2$.

(iii) $\Rightarrow$ (ii). We assume that $\operatorname{Cir}(\Gamma)$ is connected, and we will show that $\Lambda^{1}(\Gamma)$ is indecomposable. For this, as $\Lambda^{1}(\Gamma)$ is spanned by vectors associated to cycles (see e.g. Lemma 2), it is enough to show the following: given two oriented cycles $c^{\prime}, c^{\prime \prime}$ in $\Gamma$, there exist oriented cycles $c_{0}=c^{\prime}$, $c_{1}, \ldots, c_{k}=c^{\prime \prime}$ such that $\left\langle g_{c_{j-1}}, g_{c_{j}}\right\rangle \neq 0$ for all $j \in\{1, \ldots, k\}$.

As $\operatorname{Cir}(\Gamma)$ is connected, possibly upon changing the orientation of $c^{\prime \prime}$, we may assume without loss of generality that $c^{\prime}$ and $c^{\prime \prime}$ share at least one oriented edge, say $e_{0}$. Let $\mathbb{F} \subset \mathbb{E}$ be the set of oriented edges such that

$$
g_{c^{\prime}}+g_{c^{\prime \prime}}=2 \delta_{e_{0}}+\sum_{e \in \mathbb{F}} \alpha_{e} \delta_{e}
$$

with $\alpha_{e} \in\{1,2\}$ for all $e \in \mathbb{F}$. There are also oriented cycles $c_{1}, \ldots, c_{m}$ with support in $\mathbb{F} \cup\left\{e_{0}\right\}$ such that

$$
g_{c^{\prime}}+g_{c^{\prime \prime}}=g_{c_{1}}+\cdots+g_{c_{m}}
$$

and we may assume that $c_{1}$ contains $e_{0}$. Both $\left\langle g_{c^{\prime}}, g_{c_{1}}\right\rangle$ and $\left\langle g_{c^{\prime \prime}}, g_{c_{1}}\right\rangle$ are sums over $e_{0} \cup \mathbb{F}$ of integers which are all $\geq 0$, with the integers corresponding to $e_{0}$ being $>0$. Hence

$$
\left\langle g_{c^{\prime}}, g_{c_{1}}\right\rangle \neq 0 \neq\left\langle g_{c^{\prime \prime}}, g_{c_{1}}\right\rangle
$$

and this ends the proof.

An analogous result holds for the lattice $N^{1}(\Gamma)$.

Lemma 4. - Let $\Gamma$ be a connected graph. Then the following are equivalent:

(i) $\Gamma$ is separable,

(ii) $N^{1}(\Gamma)$ is decomposable.

\section{Proof.}

(i) $\Rightarrow$ (ii). Let $v_{0} \in V$ be a cut vertex and let $\Gamma_{1}, \ldots, \Gamma_{b}$ be the connected components of the subgraph of $\Gamma$ induced by $V \backslash\left\{v_{0}\right\}$. For $j \in\{1, \ldots, b\}$, let $V_{j}$ denote the vertex set of $\Gamma_{j}$; observe that $V_{1} \amalg \ldots \amalg V_{b}=V \backslash\left\{v_{0}\right\}$. Then $\left(d\left(\delta_{v}\right)\right)_{v \in V_{1} \amalg \ldots \amalg V_{b}}$ is a basis of $N^{1}(\Gamma)$ and $\left\langle d\left(\delta_{v}\right), d\left(\delta_{w}\right)\right\rangle=0$ if $v \in V_{j}$, $w \in V_{k}, j \neq k$. It follows that $N^{1}(\Gamma)$ is an orthogonal sum of factors, one for each block of $\Gamma$.

TOME $125-1997-\mathrm{N}^{\circ} 2$ 
(ii) $\Rightarrow$ (i). Let $N^{1}(\Gamma)=N^{\prime} \oplus N^{\prime \prime}$ be a non trivial orthogonal decomposition. Let $b$ be an oriented bond in $\Gamma$. Writing $g_{b}=\left(g^{\prime}, g^{\prime \prime}\right) \in N^{\prime} \oplus N^{\prime \prime}$, one has either $g^{\prime \prime}=0$ or $g^{\prime}=0$. Indeed, as

$$
\left\langle g_{b}, g_{b}\right\rangle=\left\langle g_{b}^{\prime}, g_{b}^{\prime}\right\rangle+\left\langle g_{b}^{\prime \prime}, g_{b}^{\prime \prime}\right\rangle
$$

is the number of edges in $b$, the support of $b$ is the disjont union of the support of $b^{\prime}$ and of that of $b^{\prime \prime}$. One concludes by minimality of $b$ that $g_{b}=g_{b}^{\prime}$ or $g_{b}=g_{b}^{\prime \prime}$.

Consider now the cuts $d\left(\delta_{v}\right)$ for $v \in V$. If all of them were bonds, we could define $V^{\prime}=\left\{v \in V \mid d\left(\delta_{v}\right) \in N^{\prime}\right\}$, and similarly for $N^{\prime \prime}$, and the corresponding induced subgraphs would be disconnected from each other, in contradiction with the hypothesis. Hence there exists at least one vertex $v_{0} \in V$ such that $d\left(\delta_{v_{o}}\right)$ is not a bond. Then $d\left(\delta_{v_{o}}\right)$ properly contains some bond, hence $v_{0}$ is a cut vertex of $\Gamma$, so that $\Gamma$ is separable.

Proposition 4 is a rephrasement of Lemmas 3 and 4.

Proof of Proposition 5.

There is no loss of generality if we assume that $\Gamma$ and $\Gamma^{\prime}$ are obtained as follows. Given a graph $\Gamma_{1}$ with two distinct vertices $u_{1}, v_{1}$ and a graph $\Gamma_{2}$ with two distinct vertices $u_{2}, v_{2}$, the graph $\Gamma$ is obtained from the disjoint union $\Gamma_{1} \amalg \Gamma_{2}$ by the identifications

$$
u_{1}=u_{2}=u \quad \text { and } \quad v_{1}=v_{2}=v
$$

and the graph $\Gamma^{\prime}$ is obtained similarly by the identifications

$$
u_{1}=v_{2}=u^{\prime} \quad \text { and } \quad v_{1}=u_{2}=v^{\prime} .
$$

According to the definition in Section $2, \Gamma^{\prime}$ is obtained from $\Gamma$ by twisting about $\{u, v\}$.

For each $\lambda \in \Lambda^{1}(\Gamma)$, define $\lambda^{\prime} \in C^{1}\left(\Gamma^{\prime}, \mathbb{Z}\right)$ by

$$
\lambda^{\prime}(e)=\left\{\begin{aligned}
\lambda(e) & \text { if } e \in \mathbb{E}\left(\Gamma_{1}\right) \\
-\lambda(e) & \text { if } e \in \mathbb{E}\left(\Gamma_{2}\right)
\end{aligned}\right.
$$

We claim that $\lambda^{\prime} \in \Lambda^{1}\left(\Gamma^{\prime}\right)$; it follows that the application $\lambda \mapsto \lambda^{\prime}$ is an isomorphism $\Lambda^{1}(\Gamma) \stackrel{\approx}{\longrightarrow} \Lambda^{1}\left(\Gamma^{\prime}\right)$. The claim means that $\left(d^{*} \lambda^{\prime}\right)(v)=0$ for all $v \in V\left(\Gamma^{\prime}\right)$; the non trivial equalities in the claim are

$$
\left(d^{*} \lambda^{\prime}\right)\left(u^{\prime}\right)=0=\left(d^{*} \lambda^{\prime}\right)\left(v^{\prime}\right)
$$


As $\lambda \in \Lambda^{1}(\Gamma)$, one has

$$
\left(d^{*} \lambda\right)(w)=\sum_{\substack{e \in \mathbb{E}(\Gamma) \\ e_{+}=w}} \lambda(e)=0
$$

for all $w \in V(\Gamma)$, where $e_{+}, e_{-}$denote respectively the head and the tail of $e$. In particular

$$
\begin{aligned}
& \sum_{\substack{e \in \mathbb{E}\left(\Gamma_{1}\right) \\
e_{+}=u_{1}}} \lambda(e)=\sum_{\substack{e \in \mathbb{E}\left(\Gamma_{2}\right) \\
e=u_{2}}} \lambda(e), \\
& \sum_{\substack{e \in \mathbb{E}\left(\Gamma_{1}\right) \\
e-=v_{1}}} \lambda(e)=\sum_{\substack{e \in \mathbb{E}\left(\Gamma_{2}\right) \\
e_{+}=v_{2}}} \lambda(e), \\
& \sum_{\substack{e \in \mathbb{E}\left(\Gamma_{2}\right) \\
e_{-}=u_{2}}} \lambda(e)=\sum_{\substack{e \in \mathbb{E}\left(\Gamma_{2}\right) \\
e_{+}=v_{2}}} \lambda(e)
\end{aligned}
$$
(the last equality is easily obtained from $\left.\sum_{w \in V\left(\Gamma_{1}\right)}\left(d^{*} \lambda\right)(w)=0\right)$. It follows
that one has also

$$
\begin{aligned}
d^{*} \lambda^{\prime}\left(u^{\prime}\right) & =\sum_{\substack{e \in \mathbb{E}\left(\Gamma_{1}\right) \\
e_{+}=u_{1}}} \lambda^{\prime}(e)+\sum_{\substack{e \in \mathbb{E}\left(\Gamma_{2}\right) \\
e_{+}=v_{2}}} \lambda^{\prime}(e) \\
& =\sum_{\substack{e \in \mathbb{E}\left(\Gamma_{1}\right) \\
e_{+}=u_{1}}} \lambda(e)-\sum_{\substack{e \in \mathbb{E}\left(\Gamma_{2}\right) \\
e_{+}=v_{2}}} \lambda(e)=0
\end{aligned}
$$

and similarly $\left(d^{*} \lambda^{\prime}\right)\left(v^{\prime}\right)=0$, so that the claim is proved.

For each $\nu \in N^{1}(\Gamma)$, define $\nu^{\prime} \in C^{1}\left(\Gamma^{\prime}, \mathbb{Z}\right)$ by

$$
\nu^{\prime}(e)=\left\{\begin{aligned}
\nu(e) & \text { if } e \in \mathbb{E}\left(\Gamma_{1}\right), \\
-\nu(e) & \text { if } e \in \mathbb{E}\left(\Gamma_{2}\right) .
\end{aligned}\right.
$$

We claim that $\nu^{\prime} \in N^{1}\left(\Gamma^{\prime}\right)$; it follows that the application $\nu \mapsto \nu^{\prime}$ is an isomorphism $N^{1}(\Gamma) \stackrel{\approx}{\longrightarrow} N^{1}\left(\Gamma^{\prime}\right)$.

To prove the claim, it is enough to check that $\nu^{\prime}$ is orthogonal to $\Lambda^{1}\left(\Gamma^{\prime}\right)$. But any element of $\Lambda^{1}\left(\Gamma^{\prime}\right)$ is the image $\lambda^{\prime}$ of some $\lambda \in \Lambda^{1}(\Gamma)$ by the isomorphism above. Thus

$$
\left\langle\nu^{\prime}, \lambda^{\prime}\right\rangle=\langle\nu, \lambda\rangle=0
$$

and the claim is proved. 


\section{On Voronoi polyhedra: proof of Proposition 6}

As announced in Section 2, we characterize here the codimension-onefaces of the Voronoi polyhedra

$$
\begin{aligned}
& \operatorname{Vor}_{\Lambda^{1}(\Gamma)}=\left\{x \in \operatorname{Ker}\left(\Delta_{1}\right) \mid\langle x, x\rangle \leq\langle x-\lambda, x-\lambda\rangle\right. \\
& \left.\qquad \text { for all } \lambda \in \Lambda^{1}(\Gamma) \backslash\{0\}\right\}
\end{aligned}
$$

and $\operatorname{Vor}_{N^{1}(\Gamma)}$.

Recall that a vector $\lambda \neq 0$ in a lattice $\Lambda \subset \mathcal{H}$ is called relevant if the affine hyperplane

$$
\{x \in \mathcal{H} \mid\langle x, x\rangle=\langle x-\lambda, x-\lambda\rangle\}
$$

contains a codimension-one-face of the Voronoi polyhedron of $\Lambda$. For the following lemma, we agree that a vector $\lambda \in \Lambda$ is reduced if $\langle\lambda, \lambda\rangle \leq\left\langle\lambda^{\prime}, \lambda^{\prime}\right\rangle$ for all $\lambda^{\prime} \in \lambda+2 \Lambda$.

Lemma 5. - $A$ vector $\lambda \in \Lambda \backslash\{0\}$ is relevant if and only if $\lambda$ is reduced and $\lambda,-\lambda$ are the only reduced vectors in $\lambda+2 \Lambda$.

Proof. - See Theorem 10, Chap. 21 of the second edition of [CoS].

Consider a connected graph $\Gamma$ and the associated lattices

$$
\Lambda^{1}(\Gamma) \subset \operatorname{Ker}\left(\Delta_{1}\right) \text { and } N^{1}(\Gamma) \subset \operatorname{Im}(d)
$$

\section{Proof of Proposition 6.}

Claim (i). Let $c$ be an oriented circuit of $\Gamma$. As $g_{c}$ takes values in $\{-1,0,1\}$, any $\lambda^{\prime} \in g_{c}+2 \Lambda^{1}(\Gamma)$ takes even values outside the edge-set of $c$ and odd values on the edge-set of $c$. If moreover $\lambda^{\prime} \neq \pm g_{c}$ there exists $e \in \mathbb{E}$ such that $\left|\lambda^{\prime}(e)\right|>\left|g_{c}(e)\right|$, so that $\left\langle\lambda^{\prime}, \lambda^{\prime}\right\rangle>\left\langle g_{c}, g_{c}\right\rangle$. It follows from Lemma 5 that $g_{c}$ is relevant.

Conversely, let $\lambda \in \Lambda^{1}(\Gamma)$ be a relevant vector; we have to show that there exists a circuit $c$ in $\Gamma$ such that $\lambda=g_{c}$. As $\lambda \neq 0$ there exists an oriented edge $e_{1} \in \mathbb{E}$ such that $\lambda\left(e_{1}\right) \geq 1$. As $\lambda$ is a flow, there exists an oriented circuit $c$ with cyclically ordered edges $e_{1}, e_{2}, \ldots, e_{k}, e_{k+1}=e_{1}$ such that $\lambda\left(e_{j}\right) \geq 1$ for all $j \in\{1, \ldots, k\}$; in particular

$$
\left\langle\lambda, g_{c}\right\rangle \geq k=\left\langle g_{c}, g_{c}\right\rangle
$$

On the other hand

$$
\left\langle\lambda-2 g_{c}, \lambda-2 g_{c}\right\rangle=\langle\lambda, \lambda\rangle+4\left\langle g_{c}, g_{c}\right\rangle-4\left\langle\lambda, g_{c}\right\rangle \geq\langle\lambda, \lambda\rangle
$$


because $\lambda$ is reduced. It follows that

$$
\left\langle\lambda, g_{c}\right\rangle=k
$$

and that $\lambda(e) \in\{-1,0,1\}$ for all $e \in \mathbb{E}$. If one had $\lambda \neq g_{c}$, there would exist by the same argument ( $\lambda$ is a flow) another oriented circuit $c^{\prime}$ with edge-set disjoint from that of $c$, and one would have

$$
\langle\lambda, \lambda\rangle=\left\langle\lambda-2 g_{c}, \lambda-2 g_{c}\right\rangle=\left\langle\lambda-2 g_{c^{\prime}}, \lambda-2 g_{c^{\prime}}\right\rangle
$$

in contradiction with Lemma 5. Thus $\lambda=g_{c}$.

Claim (ii). If $b$ is a bond in $\Gamma$, then $g_{b}$ is relevant by the same argument as in the previous step.

Conversely, let $\nu \in N^{1}(\Gamma)$ be a relevant vector. Let $f \in C^{0}(\Gamma, \mathbb{Z})$ be such that $\nu=d f$ and set

$$
V_{0}=\{x \in V \mid f(x) \geq f(y) \text { for all } y \in V\} .
$$

Observe that $V_{0}$ depends only on $\nu$ (because $\Gamma$ is connected so that $f$ is well defined up to an additive constant) and that $V_{0}$ is a proper subset of $V$ (because $\nu \neq 0$ so that $f$ is not a constant function).

The subgraph of $\Gamma$ induced by $V_{0}$ may have several connected components. Let $\Gamma_{1}$ be one of them and let $V_{1}$ be the vertex set of $\Gamma_{1}$. We set

$$
b=\left\{e \in \mathbb{E} \mid e_{+} \in V_{1} \text { and } e_{-} \in V \backslash V_{1}\right\} .
$$

If $|b|=k$ one has

$$
\left\langle\nu, g_{b}\right\rangle \geq k=\left\langle g_{b}, g_{b}\right\rangle .
$$

The end of the argument runs as in the previous step.

\section{Proof of Propositions 7 and 8}

Proof of Proposition 7.

Claim (i). Let $v$ be a vertex of $\Gamma$. As $d\left(\delta_{v}\right) \in \operatorname{Im}(d)$ is orthogonal to $\Lambda^{1}(\Gamma)^{\sharp}$, one has

$$
\widetilde{A}\left(d\left(\delta_{v}\right)\right)=\sum_{\substack{e \in \mathbb{E} \\ e_{+}=v}} \widetilde{A}\left(\delta_{e}\right)=0 .
$$

Choose an oriented path $\gamma$ from $v_{0}$ to $v$. Join $v_{0}$ to each $w \sim v$ by an oriented path $\gamma_{w}$ which begins by $\gamma$ and which ends by an edge from $v$ to $w$. Then

$$
\operatorname{deg}(v) \widetilde{A}(\gamma)-\sum_{\substack{w \in V \\ w \sim v}} \widetilde{A}\left(\gamma_{w}\right)=-\sum_{\substack{e \in \mathbb{E} \\ e_{-}=v}} \widetilde{A}\left(\delta_{e}\right)=\sum_{\substack{e \in \mathbb{E} \\ e_{+}=v}} \widetilde{A}\left(\delta_{e}\right)=0
$$

TOME $125-1997-\mathrm{N}^{\circ} 2$ 
and consequently

$$
\operatorname{deg}(v) A_{v_{0}}-\sum_{\substack{w \in V \\ w \sim v}} A_{v_{0}}(w)=0
$$

In other words, $A_{v_{0}}$ is harmonic.

The image of $A_{v_{0}}$ generates the Jacobian of $\Gamma$, as has already been observed just after the definition of the Jacobian.

Claim (ii). If $\Gamma$ is a polygon with $n$ vertices and $n$ edges, its Jacobian $\left(\Lambda^{1}(\Gamma)\right)^{\sharp} / \Lambda^{1}(\Gamma)$ is a cyclic group of order $n$. As $A_{v_{0}}$ is clearly surjective, it is a bijection.

Claim (iii). By (i) and by the observation of Section 3 which precedes Proposition 7, there exists a group homomorphism

$$
\alpha: \operatorname{Pic}(\Gamma) \longrightarrow \mathcal{J}(\Gamma)
$$

which maps the class of $v-v_{0}$ to $A_{v_{0}}(v)$ for each $v \in V$ and which is onto.

We have already observed in Section 3 that the linear map

$$
d^{*}: C^{1}(\Gamma, \mathbb{R}) \longrightarrow \operatorname{Im}\left(d^{*}\right)
$$

restricts to a homomorphism from $C^{1}(\Gamma, \mathbb{Z})$ onto $\operatorname{Div}^{0}(\Gamma)$. As $d^{*}$ is the composition of the orthogonal projection $\pi: C^{1}(\Gamma, \mathbb{R}) \rightarrow \operatorname{Im}(d)$ and of some linear isomorphism $\operatorname{Im}(d) \rightarrow \operatorname{Im}\left(d^{*}\right)$, the map $d^{*}$ restricts also to a homomorphism

$$
\pi\left(C^{1}(\Gamma, \mathbb{Z})\right)=\left(N^{1}(\Gamma)\right)^{\sharp} \longrightarrow \operatorname{Div}^{0}(\Gamma)
$$

which is onto (the equality holds by Lemma 1 ). As $d^{*}\left(N^{1}(\Gamma)\right)=P(\Gamma)$ by definition, one has a homomorphism

$$
\beta_{N}:\left(N^{1}(\Gamma)\right)^{\sharp} / N^{1}(\Gamma) \longrightarrow \operatorname{Div}^{0}(\Gamma) / P(\Gamma)=\operatorname{Pic}(\Gamma)
$$

which is onto. The composition of the isomorphism

$$
\phi: \mathcal{J}(\Gamma) \longrightarrow\left(N^{1}(\Gamma)\right)^{\sharp} / N^{1}(\Gamma)
$$

(see Lemma 1) and of $\beta_{N}$ provides a group homomorphism

$$
\beta_{\Lambda}: \mathcal{J}(\Gamma) \longrightarrow \operatorname{Pic}(\Gamma)
$$

which is onto.

Let $v \in V$ and let $\gamma$ be some oriented path from $v_{0}$ to $v$. As $A_{v_{0}}(v)$ is the class in $\mathcal{J}(\Gamma)$ of the orthogonal projection of $\sum_{e \in \gamma} \delta_{e}$ onto $\operatorname{Ker}\left(\Delta_{1}\right)$, the 
proof of Lemma 1 shows that $\phi\left(A_{v_{0}}(v)\right)$ is the class in $\left(N^{1}(\Gamma)\right)^{\sharp} / N^{1}(\Gamma)$ of the orthogonal projection of $\sum_{e \in \gamma} \delta_{e}$ onto $\operatorname{Im}(d)$. Hence $\beta_{\Lambda}\left(A_{v_{0}}(v)\right)$ is the class of $d^{*}\left(\sum_{e \in \gamma} \delta_{e}\right)=v-v_{0}$ in $\operatorname{Pic}(\Gamma)$, or in other terms

$$
\beta_{\Lambda}\left(A_{v_{0}}(v)\right)=P_{v_{0}}(v) \text {. }
$$

It follows that the homomorphisms $\beta_{\Lambda}$ and $\alpha$ are inverse to each other.

Claim (iv). Let $x, y$ be two distinct vertices of $\Gamma$. As $\Gamma$ is nonseparable, there exists an oriented circuit $c$ which contains $x$ and $y$. (This is a consequence of Menger's theorem; see e.g. [VLW, p. 407].) Let $n$ be the size of $c$ and let

$$
v_{1}=x, v_{2}, \ldots, v_{k-1}, v_{k}=y, v_{k+1}, \ldots, v_{n}, v_{n+1}=v_{1}
$$

be a cyclic enumeration of the vertices of $c$. Let $h: V \rightarrow \mathbb{Z} / n \mathbb{Z}$ be the map which applies a vertex $v \in V$ to the class of $j \in \mathbb{Z}$ if $v=v_{j}$ and to 0 otherwise. It is easy to check that $h$ is harmonic, and that $h(x) \neq h(y)$. (Observe that the map $v \mapsto h(v)-h\left(v_{0}\right)$ has the same properties and maps moreover $v_{0}$ to 0 .)

We have just shown that harmonic maps separate the vertices of $\Gamma$. It follows from the observation of Section 3 that $P_{v_{0}}: V \rightarrow \operatorname{Pic}(\Gamma)$ is injective.

Claim (iii) implies now that $A_{v_{0}}: V \rightarrow \mathcal{J}(\Gamma)$ is also an isomorphism.

Recall that a graph $\Gamma$ is planar if it embedds in the sphere. One defines the dual graph $\Gamma^{*}$ of $\Gamma$ with respect to an embedding of $\Gamma$ in the sphere. The vertices of $\Gamma^{*}$ are the components of the complement of $\Gamma$ in the sphere. There is a natural bijection $e \mapsto e^{*}$ between edges of $\Gamma$ and edges of $\Gamma^{*}$; the ends of $e^{*}$ in $\Gamma^{*}$ are the vertices corresponding to the components with which $e$ is incident. (See e.g. [VLW, Chap. 32].)

Proof of Proposition 8.

Denote by $\mathbb{E}$ the set of oriented edges of $\Gamma$ (or of $\Gamma^{*}$ ). A subset $\mathbb{F}^{\prime}$ (resp. $\mathbb{F}^{\prime \prime}$ ) of $\mathbb{E}$ is the set of some oriented circuit $c^{\prime}$ (resp. $c^{\prime \prime}$ ) in $\Gamma$ if and only if it is the set of oriented edges of some oriented bond $b^{\prime}$ (resp. $b^{\prime \prime}$ ) in $\Gamma^{*}$; moreover, if this is so, then

$$
\left\langle g_{c^{\prime}}, g_{c^{\prime \prime}}\right\rangle=\left\langle g_{b^{\prime}}, g_{b^{\prime \prime}}\right\rangle
$$

where the left-hand side is an inner product of two vectors in $\Lambda^{1}(\Gamma)$ and where the right-hand side is the inner product of the corresponding vectors in $N^{1}\left(\Gamma^{*}\right)$. As $\Lambda^{1}(\Gamma)$ has a $\mathbb{Z}$-basis $\left\{g_{c_{1}}, \ldots, g_{c_{m-n+1}}\right\}$ where $c_{1}, \ldots, c_{m-n+1}$ are oriented circuits in $\Gamma$ (e.g. by Proposition 6$)$, the vectors $\left\{g_{b_{1}}, \ldots, g_{b_{m-n+1}}\right\}$ defined by the corresponding oriented bonds in $\Gamma^{*}$ constitute a $\mathbb{Z}$-basis of $N^{1}\left(\Gamma^{*}\right)$. The two resulting Gram matrices

TOME $125-1997-\mathrm{N}^{\circ} 2$ 
being equal, the two lattices $\Lambda^{1}(\Gamma)$ and $N^{1}\left(\Gamma^{*}\right)$ are isomorphic. Similarly $N^{1}(\Gamma)$ and $\Lambda^{1}\left(\Gamma^{*}\right)$ are isomorphic. The last claim of Proposition 8 follows now from Proposition 3.

\section{Remarks and Examples}

Remarks.

1) The automorphism group $\operatorname{Aut}(\Gamma)$ of the graph $\Gamma$ acts naturally by isometries on $C^{0}(\Gamma, \mathbb{R})$ and $C^{1}(\Gamma, \mathbb{R})$, preserving $C^{0}(\Gamma, \mathbb{Z})$ and $C^{1}(\Gamma, \mathbb{Z})$ respectively. One may also check that $d$ and $d^{*}$ are equivariant operators for these actions, so that $\operatorname{Aut}(\Gamma)$ acts naturally as isometries of the lattices $\Lambda^{1}(\Gamma)$ and $N^{1}(\Gamma)$.

2) There would be no difficulty to extend all previous considerations to graphs with positive weights on vertices and edges. Inner products would then be defined by formulas such as

$$
\left\langle f_{1}, f_{2}\right\rangle=\sum_{v \in V} m(v) f_{1}(v) f_{2}(v), \quad\left\langle g_{1}, g_{2}\right\rangle=\frac{1}{2} \sum_{e \in \mathbb{E}} \ell(e) g_{1}(e) g_{2}(e)
$$

where $m: V \rightarrow \mathbb{R}_{+}^{*}$ and $\ell: E \rightarrow \mathbb{R}_{+}^{*}$ are given weights. (More generally, integral-valued negative weights would give rise to indefinite inner product spaces over $\mathbb{Z}$.)

If $(\Gamma, \ell)$ is a weighted graph with $m(v)=1$ for all $v \in V$ and $\ell(e)=\ell(\bar{e}) \in\{1,2,3, \ldots\}$ for all $e \in \mathbb{E}$, the corresponding lattice $\Lambda^{1}(\Gamma, \ell)$ is isomorphic to the lattice $\Lambda^{1}\left(\Gamma_{\ell}\right)$ where $\Gamma_{\ell}$ is obtained from $\Gamma$ by subdividing each edge $e$ in $\ell(e)$ edges. The lattice $N^{1}(\Gamma, \ell)$ in this case is isomorphic to the lattice $N^{1}\left(\Gamma^{\ell}\right)$ where $\Gamma^{\ell}$ is obtained from $\Gamma$ by replacing each edge $e$ by $\ell(e)$ edges in parallel.

3) The operators $d$ and $d^{*}$ can be identified to their matrices with respect to the bases $\left(\delta_{v}\right)_{v \in V}$ of $C^{0}(\Gamma, \mathbb{R})$ and $\left(\delta_{e}\right)_{e \in \mathbb{E}}$ of $C^{1}(\Gamma, \mathbb{R})$. As these matrices have integral entries, their reduction modulo 2 define linear maps between the spaces $C^{0}\left(\Gamma, \mathbb{F}_{2}\right)$ and $C^{1}\left(\Gamma, \mathbb{F}_{2}\right)$ of functions from $V$ and $E$ to the field of two elements.

In case $\Gamma$ is a simple connected graph, the subspace

$$
\operatorname{Im}\left(d: C^{0}\left(\Gamma, \mathbb{F}_{2}\right) \longrightarrow C^{1}\left(\Gamma, \mathbb{F}_{2}\right)\right)
$$

is known as the binary code $\mathcal{C}(\Gamma)$ of the graph $\Gamma$. In the $|E|$-dimensional space $\mathbb{F}_{2}{ }^{|E|} \approx C^{1}\left(\Gamma, \mathbb{F}_{2}\right)$, the binary code $\mathcal{C}(\Gamma)$ is the subspace generated by the columns of the matrix $d$ or by the rows of the incidence matrix $d^{*}$. (See Chap. 32 in [VLW].) 
In some sense, the lattice $N^{1}(\Gamma)$ is with respect to $\mathbb{Z}$ what the code $\mathcal{C}(\Gamma)$ is with respect to $\mathbb{F}_{2}$.

This can be generalized as follows: the lattices $N^{1}(\Gamma)$ and $\Lambda^{1}(\Gamma)$ are sublattices of $C^{1}(\Gamma, Z) \approx Z^{n}$. Fixing an orthonormal basis of $C^{1}(\Gamma, Z)$ (given by an orientation of the edge set) one gets a pair of codes

$$
C_{q}^{N}(\Gamma)=N^{1}(\Gamma) \otimes_{Z} \mathbb{F}_{q} \quad \text { and } \quad C_{q}^{\Lambda}(\Gamma)=\Lambda^{1}(\Gamma) \otimes_{Z} \mathbb{F}_{q}
$$

where $\mathbb{F}_{q}$ denotes a finite field.

Let us check that the orthogonal of $C_{q}^{N}(\Gamma)$ is precisely $C_{q}^{\Lambda}(\Gamma)$. On one hand, it is clear that $C_{q}^{\Lambda}(\Gamma) \subset C_{q}^{N}(\Gamma)^{\perp}$. On the other hand, consider $\underline{\lambda} \in C_{q}^{N}(\Gamma)^{\perp}$; let $\left(d\left(\delta_{j}\right)\right)_{1 \leq j \leq n-1}$ be the basis of $\operatorname{Im}(d)$ considered at the end of Section 5 and let $\left(\underline{h}_{j}\right)_{1 \leq j \leq n-1}$ be the corresponding basis of $C_{q}^{\Lambda}(\Gamma)$; the conditions $\underline{\lambda} \perp \underline{h}_{j}(1 \leq j \leq n-1)$ imply that $\underline{\lambda}$ is a $q$-flow on $\Gamma$; as such a $q$-flow can be represented by a $\mathbb{Z}$-flow [BrO, Prop. 6.3.7], it follows that $\underline{\lambda} \in C_{q}^{\Lambda}(\Gamma)$.

The weight enumerators of the 2 codes are obtained as specializations of Tutte's dichromatic polynomial and are related by Mac-Willams identity [BrO, Prop. 6.5.1 and 6.5.2].

Is there anything analogous relating the $\vartheta$-series of the lattices $\Lambda^{1}(\Gamma)$ and $N^{1}(\Gamma)$ ? (The obvious lattice analogue of the Mac-Williams identity is the Poisson summation formula which relates the $\vartheta$-series of a lattice and its dual lattice.)

4) The setting exposed here has a straightforward generalization to higher dimensional objects. For example, let $X$ be a finite CW-complex of dimension $d$. For each $j \in\{1, \ldots, d\}$, let $\mathbb{E}^{j}$ denote the set of oriented $j$-cells in $X$, and let $C^{j}(X, \mathbb{R})$ denote the space of all functions $f: \mathbb{E}^{j} \rightarrow \mathbb{R}$ such that $f(\bar{e})=-f(e)$ for all $e \in \mathbb{E}^{j}$ (where the bar indicates as above a change of orientation). One has a Laplacian operator

$$
\Delta_{j}=d_{j}^{*} d_{j}+d_{j-1} d_{j-1}^{*}
$$

an orthogonal decomposition

$$
C^{j}(X, \mathbb{R})=\operatorname{Ker}\left(\Delta_{j}\right) \oplus \operatorname{Im}\left(d_{j-1}\right) \oplus \operatorname{Im}\left(d_{j}^{*}\right)
$$

and lattices

$$
\left\{\begin{array}{l}
\Lambda^{j}(X)=C^{j}(X, \mathbb{Z}) \cap \operatorname{Ker}\left(\Delta_{j}\right) \subset \operatorname{Ker}\left(\Delta_{j}\right) \\
N^{j}(X)=C^{j}(X, \mathbb{Z}) \cap \operatorname{Im}\left(d_{j-1}\right) \subset \operatorname{Im}\left(d_{j-1}\right), \\
M^{j}(X)=C^{j}(X, \mathbb{Z}) \cap \operatorname{Im}\left(d_{j}^{*}\right) \subset \operatorname{Im}\left(d_{j}^{*}\right)
\end{array}\right.
$$

as above.

$$
\text { TOME } 125-1997-\mathrm{N}^{\circ} 2
$$


For example any finitely generated group

$$
G=\left\langle s_{1}, \ldots, s_{k} \mid R_{1}, \ldots, R_{\ell}\right\rangle
$$

with $k$ generators and $\ell$ relations gives rise canonically to a $C W$-complex of dimension 2 with 1 vertex, $k$ edges (which are loops) and $\ell$ faces. It would be interesting to explore the properties of the resulting lattices $\Lambda^{1}$ and $M^{1}$.

The idea to write down a "Hodge decomposition" $(*)$ in a combinatorial setting is of course most classical (see e.g. [Eck] or [Bor]), but the corresponding lattices have apparently not been systematically studied.

Here are some examples.

1. Polygons, bond graphs and root lattices. - Let $\Gamma$ be a polygon with $n$ vertices and with $n$ edges. Then $\Lambda^{1}(\Gamma) \subset \operatorname{Ker}\left(\Delta_{1}\right)$ is clearly the lattice $\sqrt{n} \mathbb{Z} \subset \mathbb{R}$ of minimal norm $n$ and determinant $n$. The Jacobian $\mathcal{J}(\Gamma)$ is the cyclic group of order $n$.

The lattice $N^{1}(\Gamma) \subset \operatorname{Im}(d)$ can be described as follows. Choose an orientation of $\Gamma$, let $e_{1}, \ldots, e_{n}$ be a cyclic enumeration of the corresponding oriented edges, and let $\delta_{j} \in C^{1}(\Gamma, \mathbb{R})$ be defined by $\delta_{j}\left(e_{j}\right)=1, \delta_{j}\left(e_{k}\right)=0$ if $k \neq j$. Then

$$
N^{1}(\Gamma)=\left\{\sum_{j=1}^{n} n_{j} \delta_{j} \mid n_{j} \in \mathbb{Z} \text { and } \sum_{j=1}^{n} n_{j}=0\right\}
$$

has a basis $\left(\delta_{j+1}-\delta_{j}\right)_{1 \leq j \leq n-1}$. This is a root lattice of type $A_{n-1}$, with minimal norm 2 and determinant $n$ (see [CoS, Chap. $4, \S 6.1]$ ). The Witt class

$$
w\left(N^{1}(\Gamma)\right)=w\left(A_{n-1}\right)=-w\left(\Lambda^{1}(\Gamma)\right)
$$

may be explicitly calculated (see [Ker, pp. 66-67]). The easiest cases are those for which $n=p^{c}$ is a prime power. If $c$ is even then $w\left(N^{1}(\Gamma)\right)=0$. If $c$ is odd then $w\left(N^{1}(\Gamma)\right)$ is not 0 , and is the class of the bilinear form

$$
b: \mathbb{Z} / n \mathbb{Z} \times \mathbb{Z} / n \mathbb{Z} \longrightarrow \mathbb{Q} / \mathbb{Z}
$$

given by $b(1,1)=n^{-1}$.

The plane dual $\Gamma^{*}$ of $\Gamma$ is the bond graph with 2 vertices and $n$ edges between them. The minimal norm of $\Lambda^{1}\left(\Gamma^{*}\right)$ is 2 .

Claim (ii) of Proposition 1 implies that a graph $\Gamma$ is simple (no loop and no multiple edge) if and only if the lattice $\Lambda^{1}(\Gamma)$ has minimal norm at least 3. 
Let $\Lambda$ be a lattice in some Euclidean space which is a root lattice in the sense that $\Lambda$ is spanned by its vectors of norm 2 . If $\Lambda$ is of the form $\Lambda^{1}(\Gamma)$ for a connected graph $\Gamma$, then $\Lambda$ is necessarily a root lattice $A_{\ell}$. Indeed, let $\alpha_{1}, \alpha_{2}$ be two distinct roots in $\Lambda^{1}(\Gamma)$. Then $\alpha_{j}$ corresponds necessarily to two edges $e_{j}, e_{j}^{\prime}$ joining the same ends $(j=1,2)$. If $\left\langle\alpha_{1}, \alpha_{2}\right\rangle \neq 0$, then the four edges $e_{1}, e_{1}^{\prime}, e_{2}, e_{2}^{\prime}$ must have the same ends. It follows that a graph with $\Lambda^{1}(\Gamma)$ a root lattice is necessarily a graph with two vertices only.

In particular, if a graph $\Gamma$ has the lattice $\Lambda^{1}(\Gamma)$ isomorphic to that of the bond graph with $n$ edges, then $\Gamma$ itself is the bond graph with $n$ edges.

2. Complete graphs. - Let $K_{n}$ be a complete graph on $n$ vertices, where $n \geq 4$. Then $\Lambda^{1}\left(K_{n}\right)$ is the "lattice of thorns", studied in [Mar] in relation with representations of the Mathieu groups $M_{12}$ and $M_{24}$.

Recall that the $n$-th lattice of thorns $T_{n}$ is defined as follows. The trivial lattice $\mathbb{Z}^{n} \subset \mathbb{R}^{n}$ gives rise by exterior power to a lattice

$$
\Lambda_{n}=\mathbb{Z}^{n} \wedge \mathbb{Z}^{n} \subset E=\mathbb{R}^{n} \wedge \mathbb{R}^{n}
$$

isomorphic to $\mathbb{Z}^{n(n-1) / 2} \subset \mathbb{R}^{n(n-1) / 2}$. If $e_{1}, \ldots, e_{n}$ is the canonical basis of $\mathbb{R}^{n}$, a lattice vector

$$
\alpha=\sum_{i<j} \alpha_{i, j} e_{i} \wedge e_{j} \in \Lambda_{n}
$$

can be viewed as a directed graph with vertex set $\{1, \ldots, n\}$ and with $\left|\alpha_{i, j}\right|$ edges between $i$ and $j$, directed from $i$ to $j$ if $\alpha_{i, j}>0$ and from $j$ to $i$ if $\alpha_{i, j}<0$. Let $F \subset E$ be the subspace of $E$ of dimension $\frac{1}{2}(n-1)(n-2)$ defined by any $(n-1)$ among the $n$ equations

$$
\left\{\begin{array}{l}
\alpha_{1,2}+\alpha_{1,3}+\cdots+\alpha_{1, n}=0 \\
\cdots \\
\alpha_{n, 1}+\alpha_{n, 2}+\cdots+\alpha_{n, n-1}=0
\end{array}\right.
$$

(with $\alpha_{j, i}=-\alpha_{i, j}$ if $j>i$ ). The $n$-th thorns lattice is by definition the lattice

$$
T_{n}=\Lambda_{n} \cap F \subset F .
$$

A lattice vector $\alpha=\sum_{i<j} \alpha_{i, j} e_{i} \wedge e_{j} \in T_{n}$ can be viewed as a zero-sum oriented graph on vertices $\{1, \ldots, n\}$, where "zero-sum" means that the indegree and the outdegree of any vertex are equal.

Let $T_{i, j, k}$ denote a "thorn" $\{i, j, k\}$, namely a triangle on the vertices $\{i, j, k\}$ with

$$
\alpha_{i, j}=\alpha_{j, k}=\alpha_{k, i}=1 .
$$

Then $\left(T_{1, i, j}\right)_{1<i<j \leq n}$ is a $\mathbb{Z}$-basis of the lattice $T_{n}$. This basis is isomorphic to the basis of the lattice $\Lambda^{1}\left(K_{n}\right)$ associated to the spanning tree $T$ in $K_{n}$ where the edge-set of $T$ consists of $(n-1)$ edges adjacent to one vertex.

TOME $125-1997-\mathrm{N}^{\circ} 2$ 
The minimal norm of the lattice $T_{n}$ is 3 (the girth of $K_{n}$ ) and the determinant is $n^{n-2}$ (the complexity of $\left.K_{n}\right)$. The dual lattice $\left(\Lambda^{1}\left(K_{n}\right)\right)^{\sharp}$ is explicitly described in [Mar], and one could deduce from that description the structure of the Jacobian of $K_{n}$. But we find it easier to turn to the Picard group.

Let $\left\{v_{1}, \ldots, v_{n}\right\}$ be an enumeration of the vertices of $K_{n}$, and denote by $\delta_{j}$ the characteristic function of $v_{j}(1 \leq j \leq n)$. The group $P\left(K_{n}\right)$ of principal divisors is generated by the elements

$$
(n-1) \delta_{j}-\sum_{\substack{1 \leq k \leq n \\ k \neq j}} \delta_{k}=\sum_{k=1}^{n} \Delta_{j, k} \delta_{k}
$$

for $j \in\{1, \ldots, n\}$, where $\Delta_{0}=\left(\Delta_{j, k}\right)_{1 \leq j, k \leq n}$ is the $n \times n$ matrix of the Laplacian. A routine computation shows that there exist matrices $U$ and $V$ in $\operatorname{GL}(n, \mathbb{Z})$ such that

$$
U \Delta_{0} V=\left(\begin{array}{cccccc}
1 & 0 & 0 & \ldots & 0 & 0 \\
0 & n & 0 & \ldots & 0 & 0 \\
0 & 0 & n & \ldots & 0 & 0 \\
\vdots & \vdots & \vdots & \ddots & \vdots & \vdots \\
0 & 0 & 0 & \ldots & n & 0 \\
0 & 0 & 0 & \ldots & 0 & 0
\end{array}\right)
$$

(see e.g. [Bou, p. A.VII.21]). It follows that

$$
\operatorname{Pic}\left(K_{n}\right) \approx \mathcal{J}\left(K_{n}\right) \approx(\mathbb{Z} / n \mathbb{Z})^{n-2} .
$$

Computations show that the corresponding Witt class is sometimes zero, for example when $n$ is a square, and sometimes not zero.

An element of the lattice $N^{1}\left(K_{n}\right)$ may be viewed as an oriented graph on vertices $\{1, \ldots, n-1\}$, such that each vertex is either the head or the tail of all edges incident to it. Let $C^{i}, 1 \leq i \leq n-1$ denote a "claw" $\{i\}$, namely an oriented graph with $\alpha_{i, j}=1$ for each $j \neq i$ and $\alpha_{k, \ell}=0$ for $k \neq i$ and for any $\ell$. Then $\left(C^{i}\right)_{1<i<n-1}$ is a $\mathbb{Z}$-basis of the lattice $N^{1}\left(K_{n}\right)$.

The number of non-oriented bonds in $K_{n}$ is the number of non-trivial partitions of the vertex set of $K_{n}$, namely

$$
\frac{1}{2}\left\{\left(\begin{array}{l}
n \\
1
\end{array}\right)+\cdots+\left(\begin{array}{c}
n \\
n-1
\end{array}\right)\right\}=2^{n-1}-1 .
$$

It follows from Proposition 6.ii that the Voronoi cell of $N^{1}\left(K_{n}\right)$ has $\left(2^{n-1}-1\right)$ pairs of parallel faces. Now it is known that, for any lattice in 
a Euclidean space of dimension $(n-1)$, the number $p$ of pairs of parallel faces of the Voronoi cell satisfies the inequalities

$$
n-1 \leq p \leq 2^{n-1}-1
$$

(the first inequality is trivial, the second one is classical - see Thm. 10 of Chap. 21 in the second edition of [CoS]). Thus the lattice $N^{1}\left(K_{n}\right)$ is one for which the second inequality above is an equality.

3. Strongly regular graphs. - Let $\Gamma$ be a connected strongly regular graph with parameters $(n, k, \lambda, \mu)$. There are classical formulas for the eigenvalues and their multiplicities of the Laplacian $\Delta_{0}$, so that the order of the group $\mathcal{J}(\Gamma)$ is easy to compute (see e.g. [Bi1]). But the actual structure of $\mathcal{J}(\Gamma)$ requires further computations.

If $P e t$ is the Petersen graph with parameters $(10,3,0,1)$, the eigenvalues of $\Delta_{0}$ are $0,2,5$ (resp. with multiplicities $1,5,4$ ), so that

$$
\mid \mathcal{J}(\text { Pet }) \mid=\frac{2^{5} \times 5^{4}}{10}=2000 .
$$

The computation of the invariant factors of $\Delta_{0}$ (see the analogous formula for $U \Delta_{0} V$ in the previous example) shows that

$$
\mathcal{J}(\text { Pet }) \approx \mathbb{Z} / 2 \mathbb{Z} \oplus(\mathbb{Z} / 10 \mathbb{Z})^{3}
$$

There are two non-isomorphic strongly regular graphs with parameters $(16,6,2,2)$, which are switching equivalent. Eigenvalues computations show that their Jacobian has order

$$
\frac{4^{6} \times 8^{9}}{16}=2^{35}
$$

The first of these is the lattice graph $L(4)$, for which one has

$$
\mathcal{J}(L(4)) \approx(\mathbb{Z} / 8 \mathbb{Z})^{5} \oplus(\mathbb{Z} / 32 \mathbb{Z})^{4} .
$$

(For this computations and all the next ones, we are most grateful to Shalom Eliahou, who has used the function ismith of Maple V. Computations which are quite similar are reported in [BVE].) The other one is the so-called Shrikhande graph (see [BrR, e.g. Fig. 5.2]), for which

$$
\mathcal{J}(\mathrm{Shr}) \approx(\mathbb{Z} / 2 \mathbb{Z}) \oplus(\mathbb{Z} / 8 \mathbb{Z})^{2} \oplus(\mathbb{Z} / 16 \mathbb{Z})^{2} \oplus(\mathbb{Z} / 32 \mathbb{Z})^{4} .
$$

Thus the structure of $\mathcal{J}(\Gamma)$ for a strongly regular graph does not depend on the parameters (or on the spectrum) only. Similarly, there are four nonisomorphic strongly regular graphs with parameters $(28,12,6,4)$, which

TOME $125-1997-\mathrm{N}^{\circ} 2$ 
are switching equivalent. The Jacobian of the triangular graph $T(8)$ is the direct sum of cyclic groups of orders 2 (multiplicity 2), 14 (multiplicity 13) and 112 (multiplicity 6). For each of the other three, the so-called Chang graphs, the Jacobian is the direct sum of cyclic groups of orders 14 (multiplicity 12), 56 (multiplicity 1) and 112 (multiplicity 6).

For each prime power $q$ of the form $4 k+1$, the Paley graph $\Gamma_{q}$ has parameters

$$
\left(q, \frac{1}{2}(q-1), \frac{1}{4}(q-5), \frac{1}{4}(q-1)\right)
$$

and the order of its Jacobian is

$$
\left|\mathcal{J}\left(\Gamma_{q}\right)\right|=\frac{1}{q}\left(\frac{q-\sqrt{q}}{2}\right)^{(q-1) / 2}\left(\frac{q+\sqrt{q}}{2}\right)^{(q-1) / 2}=\frac{1}{q}\left(\frac{q^{2}-q}{4}\right)^{(q-1) / 2} .
$$

A computation shows that, for $q=5,13,17,29,37,41$,

$$
\mathcal{J}\left(\Gamma_{q}\right) \approx\left(\mathbb{Z} / \frac{1}{4}(q-1) \mathbb{Z}\right) \oplus\left(\mathbb{Z} / \frac{1}{4} q(q-1) \mathbb{Z}\right)^{(q-3) / 2} .
$$

At the time of writing, we have not found a pair of 3-connected nonisomorphic graphs with isomorphic $\Lambda^{1}(\Gamma)$.

\section{BIBLIOGRAPHIE}

[Ber] Berman (K.A.). - Bicycles and Spanning Trees, SIAM J. Alg. Disc. Meth., t. 7, 1986, p. 1-12.

[Bi1] Biggs (N.). - Algebraic Graph Theory. - Cambridge University Press, 1974 (Second Edition 1993).

[Bi2] Biggs (N.). - Homological Coverings of Graphs, J. London Math. Soc (2), t. 30, 1984, p. 1-14.

[Bi3] Biggs (N.). - The Potential of Potential Theory, Preprint, London School of Economics, 1994, Preprint to appear in the volume "The Future of Graph Theory".

[Bi4] Biggs (N.). - Algebraic Potential Theory on Graphs, Bull. London Math. Soc., to appear.

[Bol] Bollobas (B.). - Extremal Graph Theory. - Academic Press, 1978.

[Bor] Borel (A.). - Cohomologie de certains groupes discrets et laplacien p-adique, Séminaire Bourbaki, t. 437, 1973/1974. 
[Bou] Bourbaki (N.). - Algèbre, chapitres 4 à 7. - Masson, 1981.

[BCN] Brouwer (A.E.), Cohen (A.M.) and Neumaier (A.). - Distance Regular Graphs. - Springer-Verlag, 1989.

[BVE] Brouwer (A.E.) and VAn EiJl (C.A.). - On the p-rank of the adjacency matrices of strongly regular graphs, J. Alg. Combinatorics, t. 1, 1992, p. 329-346.

[BrR] Brualdi (R.) and Ryser (H.J.). - Combinatorial Matrix Theory. - Cambridge Univ. Press, 1991.

[BrO] Brylawski (T.) and J. Oxley. - The Tutte Polynomial and its Applications, in "Matroid Applications", N. White Ed., Cambridge University Press, 1992, p. 123-225.

[CoS] Conway (J.H.) and N.J.A. Sloane. - Sphere Packings, Lattices and Groups. - Springer-Verlag, 1991.

[CDS] CvetKovic (D.M.), Doob (M.) and SaChs (H.). - Spectra of Graphs. - Academic Press, 1980.

[Dix] Dixmier (J.). - Les $C^{*}$-algèbres et leurs représentations, $2^{\mathrm{e}}$ édition. - Gauthier-Villars, 1969.

[Eck] Eckmann (B.). - Harmonische Funktionen und Radwertaufgaben in einem Komplex, Comment. Math. Helv., t. 17, 1944/1945, p. 240-255.

[Ker] Kervaire (M.). - Unimodular Lattices with a Complete Root System, L'Enseignement mathématique, t. 40, 1994, p. 59-104.

[Mar] Margolin (R.S.). - Representations of $M_{12}$, J. Algebra, t. 156, 1993 , p. 362-369.

[Nar] Narasimhan (R.). - Compact Riemann Surfaces. - Birkhäuser, 1992.

[OdS] Oda (T.), Seshadri (C.S.). - Compactification of the Generalized Jacobian Variety, Trans. AMS, t. 253, 1979, p. 1-90.

[Oxl] OxLey (J.G.). - Matroid Theory. - Oxford University Press, 1992.

[Rot] Rotman (J.J.). - An Introduction to Algebraic Topology. Springer, 1988.

[Sch] Scharlau (W.). - Quadratic and Hermitian Forms. - SpringerVerlag, 1985 .

[Ser] Serre (J.-P.). - Arbres, amalgames, $\mathrm{SL}_{2}$. - Astérique, t. 46, Soc. Math. France, 1977 .

[VLW] van Lint (J.H.) and Wilson (R.M.). - A Course in Combinatorics. - Cambridge University Press, 1992.

TOME $125-1997-\mathrm{N}^{\circ} 2$ 